\title{
Entanglement Spreading in a Minimal Model of Maximal Many-Body Quantum Chaos
}

\author{
Bruno Bertini, Pavel Kos, and Tomaž Prosen \\ Department of Physics, Faculty of Mathematics and Physics, University of Ljubljana, \\ Jadranska 19, SI-1000 Ljubljana, Slovenia
}

(Received 20 December 2018; published 17 May 2019)

\begin{abstract}
The spreading of entanglement in out-of-equilibrium quantum systems is currently at the center of intense interdisciplinary research efforts involving communities with interests ranging from holography to quantum information. Here we provide a constructive and mathematically rigorous method to compute the entanglement dynamics in a class of "maximally chaotic," periodically driven, quantum spin chains. Specifically, we consider the so-called "self-dual" kicked Ising chains initialized in a class of separable states and devise a method to compute exactly the time evolution of the entanglement entropies of finite blocks of spins in the thermodynamic limit. Remarkably, these exact results are obtained despite the maximally chaotic models considered: Their spectral correlations are described by the circular orthogonal ensemble of random matrices on all scales. Our results saturate the so-called "minimal cut" bound and are in agreement with those found in the contexts of random unitary circuits with infinite-dimensional local Hilbert space and conformal field theory. In particular, they agree with the expectations from both the quasiparticle picture, which accounts for the entanglement spreading in integrable models, and the minimal membrane picture, recently proposed to describe the entanglement growth in generic systems. Based on a novel "duality-based" numerical method, we argue that our results describe the entanglement spreading from any product state at the leading order in time when the model is nonintegrable.

DOI: 10.1103/PhysRevX.9.021033

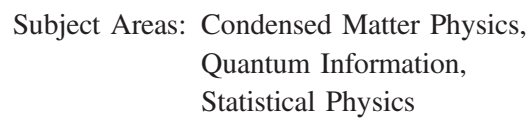

\section{INTRODUCTION}

Entanglement is arguably the most distinctive feature of quantum mechanics. It generates special kinds of nonlocal correlations which can be present in quantum states but have no analogues in the classical realm. While its elusive nature puzzled physicists for many years [1,2], it is currently regarded as a powerful resource for advances both in technological applications and in the theoretical understanding of the physical world. In particular, it plays a crucial role in the study of quantum many-body systems out of equilibrium $[3,4]$. This is due to two main reasons. First, the growth of entanglement during the nonequilibrium dynamics measures the increasing complexity of a timeevolving quantum state, with immediate implications on the feasibility of tensor network simulations [5-9]. Second, the evolution of the entanglement gives crucial information on how equilibrium statistical mechanics emerges from many-body quantum dynamics. Specifically, it is now

Published by the American Physical Society under the terms of the Creative Commons Attribution 4.0 International license. Further distribution of this work must maintain attribution to the author(s) and the published article's title, journal citation, and DOI. understood that the thermodynamic entropy of the statistical ensemble describing local observables at infinite times is a measure of the entanglement accumulated during the time evolution [10-14].

Moreover, the very way in which the entanglement spreads for finite times appears to be among the most universal aspects of many-body dynamics. Consider for instance an initial separable state, where none of the local constituents is entangled with any other. Switching on spatially local Hamiltonian interactions throughout the system (a procedure called "global quench"), one finds quite generally that the bipartite entanglement between a large connected region of the system and the rest grows linearly in time. This scenario has been observed in a large number of analytical and numerical investigations [15-44] and recently even in cold atomic experiments [45]. Known exceptions to this empirical fact are systems exhibiting realspace localization [16,46], confinement [47], and quenched disorder creating weak links [48]. In particular, the logarithmic spreading of entanglement in the presence of manybody localization (MBL) [49] is one of the main defining features of the MBL phase.

The linear growth of entanglement after a global quench has been first observed in the context of $(1+1)$ dimensional conformal field theory (CFT), where it has 
been explained in terms of an intuitive quasiparticle picture [15]. The initial state, which is not an eigenstate of the Hamiltonian, can be thought of as a collection of pairs of oppositely moving quasiparticles. These, in the course of time, spread the entanglement across the system in a similar way as the one conceived in the historical gedanken experiment by Einstein et al. [1]. In this picture, the entanglement between two different portions of the system is given by the number of pairs sharing a particle with each portion. The same idea can be used to explain the entanglement spreading in systems with stable quasiparticle excitations, for instance, free [17] and interacting [26] integrable models. It does not account, however, for the linear growth of entanglement observed in systems with no identifiable quasiparticle content such as generic interacting systems [42-44] or holographic CFTs [33-36].

More recently, a fruitful avenue of research came from the study of the so-called random quantum circuits [48,50-55], where the dynamics is completely random in space, and the only constraint is given by the locality of interactions. In this case, the linear growth of entanglement can be explained using a "minimal membrane" picture [50,53], which is conjectured to apply, at least at the qualitative level, to generic (nonintegrable) clean and noisy systems in any spatial dimension. In essence, one quantifies the amount of entanglement between two portions of the system by measuring the surface of the minimal space-time membrane separating the two portions. This picture has been analytically tested in certain limiting regimes of random quantum circuits, specifically assuming that the Hilbert space dimension $q$ per local constituent is large $(q \gg 1)$. Results are available both when the dynamics are random also in time [50,54] and when they are periodically driven [55]. No analytical result, however, exists on entanglement dynamics in specific nonintegrable models with local interactions and small finite $q$ or, in general, for clean systems.

In this paper, we fill this gap providing exact results for the entanglement dynamics of quantum-chaotic spin chains with two-dimensional local Hilbert space $(q=2)$. Specifically, we consider a family of Floquet-Ising chains which undergo a transition between integrability and ergodicity (or quantum chaos) by turning on a longitudinal magnetic field. The latter may be either spatially homogeneous or arbitrarily spatially modulated. Note that the nonintegrability of the model for nonvanishing longitudinal magnetic fields has recently been proved by computing exactly the spectral statistics [56]. We identify a class of separable initial states, homogeneous or arbitrarily modulated in space, from which the entanglement dynamics can be computed exactly for any nondisjoint bipartition. These results are of high significance for three main reasons. (i) They provide an exact verification of both the linear growth of entanglement and its relaxation to the thermodynamic entropy in concrete quantum-chaotic models. (ii) They provide a general method allowing one to obtain exact results for the nonequilibrium dynamics of manybody quantum systems even in the absence of integrability. (iii) They are valid in both the integrable and the nonintegrable cases, allowing for a unified interpretation of the entanglement spreading.

The paper is laid out as follows. In Sec. II we present the model considered, define the protocol used to drive it out of equilibrium, and introduce the entanglement measures of interest. In Sec. III we present a comprehensive summary and discussion of our results. In Sec. IV we explain the duality mapping which is the key for our analytical calculations. In Sec. V we identify the classes of initial states leading to an exactly solvable entanglement dynamics. In Sec. VI we sketch the main steps of the calculation. In Sec. VII we present a thorough numerical analysis of the entanglement spreading from generic separable initial states and advocate that our exact results give the leading-order-in-time description of the nonintegrable case. Finally, Sec. VIII contains our conclusions. A number of technical points and proofs are reported in the appendixes.

\section{MODEL, QUENCH PROTOCOL, AND OBSERVABLES OF INTEREST}

The main objective of this paper is to determine a minimal quantum-chaotic model [57], with local interactions and finite local Hilbert space, allowing for an exact determination of the entanglement spreading. A candidate emerging naturally in our quest is the so-called kicked Ising model [58-60], which describes a classical Ising model in the presence of a longitudinal magnetic field and periodically kicked with a transverse-magnetic field. This model is quantum chaotic in the sense that its spectral statistics are described by the circular orthogonal ensemble of random matrices [61], but, as we recently proved [56], at some specific points of its parameter space it allows for exact calculations. This is because at these points called "selfdual" points (see below), the system acquires a remarkable algebraic structure making it a maximal scrambler with local interactions.

To be more specific, let us introduce the Hamiltonian of the kicked Ising model. Setting to 1 the time interval between the kicks, we have

$$
H_{K I}[\boldsymbol{h} ; t]=H_{I}[\boldsymbol{h}]+\sum_{m=-\infty}^{\infty} \delta(t-m) H_{K},
$$

where $\delta(t)$ is the Dirac $\delta$ function, and we define

$$
\begin{gathered}
H_{I}[\boldsymbol{h}] \equiv J \sum_{j=1}^{L} \sigma_{j}^{z} \sigma_{j+1}^{z}+\sum_{j=1}^{L} h_{j} \sigma_{j}^{z}, \\
H_{K} \equiv b \sum_{j=1}^{L} \sigma_{j}^{x}
\end{gathered}
$$


In these equations, $L$ is the volume of the system, the matrices $\sigma_{j}^{a}$ with $a \in\{x, y, z\}$ are the Pauli matrices at position $j$, and we use periodic boundary conditions adopting the notation convention $\sigma_{L+1}^{a} \equiv \sigma_{1}^{a}$.

The parameters $J$ and $b$ are, respectively, the Ising coupling and the transverse "kicking" field, while the $L$-component vector $\boldsymbol{h}=\left(h_{1}, \ldots, h_{L}\right)$ describes a positiondependent longitudinal field. As anticipated before, in this paper we consider some specific points in the parameter space of the model. In particular, we focus on the self-dual points specified by the condition

$$
|J|=|b|=\frac{\pi}{4} .
$$

In Secs. IV-VI we explain how, at these points, a duality symmetry of the model allows for an analytical treatment of the entanglement dynamics. To be specific, from now on we set

$$
J=\frac{\pi}{4}, \quad b=-\frac{\pi}{4},
$$

but our results apply to all four combinations fulfilling Eq. (4). The longitudinal magnetic fields $\boldsymbol{h}$ are instead left arbitrary and are used to switch between the integrable and the nonintegrable cases. Indeed, for $\boldsymbol{h}=\mathbf{0}$ the Hamiltonian (1) is integrable (it can be mapped to a problem of noninteracting fermions), while it is ergodic (nonintegrable) for a generic choice of longitudinal fields. In the latter case, the only symmetry of Eq. (1) is time reversal. This is represented by the antiunitary operator $T$ defined by its action on the spin variables as follows:

$$
\sigma_{j}^{a} \mapsto T \sigma_{j}^{a} T=\sigma_{j}^{a *}
$$

Here, $(\cdot)^{*}$ denotes the complex conjugation in the "computational" basis composed by simultaneous eigenstates of $\left\{\sigma_{j}^{z}\right\}$ for all $j$ in $\{1,2, \ldots, L\}$,

$\mathcal{B}_{L}=\left\{|\boldsymbol{s}\rangle=\left|s_{1}, \ldots, s_{L}\right\rangle, s_{j} \in\{ \pm 1\}: \sigma_{j}^{z}|\boldsymbol{s}\rangle=s_{j}|\boldsymbol{s}\rangle\right\}$.

In this paper, we interchangeably use $s=+1 \equiv \uparrow$ and $s=$ $-1 \equiv \downarrow$ to designate the eigenvalues of Pauli matrices. We stress that, as proven in Ref. [56], the self-dual kicked Ising model is ergodic for any $\boldsymbol{h} \neq \mathbf{0}$. This means that, due to its special structure, the model never displays Floquet MBL $[49,62,63]$. Note that the special structure of the self-dual model has recently been used to design a multiparty entanglement generation algorithm [64].

The Floquet operator generated by Eq. (1) reads as

$U_{K I}[\boldsymbol{h}]=\mathfrak{I} \exp \left[-i \int_{0}^{1} d t H_{K I}[\boldsymbol{h} ; t]\right]=e^{-i H_{K}} e^{-i H_{I}[\boldsymbol{h}]}$,

where $\mathfrak{I} \exp [\cdot]$ denotes a time-ordered exponential. The time evolution generated by Eq. (8) admits a straightforward local two-qubit quantum circuit representation. This is observed by writing

$$
e^{-i H_{I}[h]}=\prod_{j \text { odd }} e^{-i(\pi / 4) \sigma_{j}^{z} \sigma_{j+1}^{z}} \prod_{j \text { even }} e^{-i(\pi / 4) \sigma_{j}^{z} \sigma_{j+1}^{z}}
$$

To drive the system out of equilibrium, we consider a global quantum quench protocol: We initialize the system in the ground state of a short-range Hamiltonian and suddenly, say, at $t=0$, we start evolving with Eq. (1). In particular, here we consider the ground states $\left|\psi_{\boldsymbol{\theta}, \boldsymbol{\phi}}\right\rangle$ of the following family of local noninteracting magnetization Hamiltonians

$$
H_{\boldsymbol{\theta}, \boldsymbol{\phi}}=-\sum_{j=1}^{L} g_{j} \vec{n}_{\theta_{j}, \phi_{j}} \cdot \vec{\sigma}_{j},
$$

where $\boldsymbol{\theta}=\left(\theta_{1}, \ldots, \theta_{L}\right)$ and $\boldsymbol{\phi}=\left(\phi_{1}, \ldots, \phi_{L}\right)$ are $L$ component vectors with components $\theta_{j} \in[0, \pi]$ and $\phi_{j} \in[0,2 \pi]$, while $g_{j}>0$ is arbitrary, and

$$
\vec{n}_{\theta, \phi}=(\sin \theta \cos \phi, \sin \theta \sin \phi, \cos \theta)
$$

is the radial unit vector in the three-dimensional space.

The ground states $\left|\psi_{\boldsymbol{\theta}, \boldsymbol{\phi}}\right\rangle$ of Eq. (10) are separable (i.e., they have zero entanglement): The spin at site $j$ points in the direction $\vec{n}_{\theta_{j}, \phi_{j}}$ of its Bloch's sphere. Namely, the states $\left|\psi_{\theta, \phi}\right\rangle$ are explicitly written as

$$
\left|\psi_{\boldsymbol{\theta}, \boldsymbol{\phi}}\right\rangle=\bigotimes_{j=1}^{L}\left[\cos \left(\frac{\theta_{j}}{2}\right)|\uparrow\rangle+\sin \left(\frac{\theta_{j}}{2}\right) e^{i \phi_{j}}|\downarrow\rangle\right] .
$$

After $t$ periods of the Floquet evolution, the state of the system then reads

$$
\left|\psi_{\boldsymbol{\theta}, \boldsymbol{\phi}}(t)\right\rangle=\left(U_{K I}[\boldsymbol{h}]\right)^{t}\left|\psi_{\boldsymbol{\theta}, \boldsymbol{\phi}}\right\rangle .
$$

We stress that this protocol is constructive and simple enough to be realizable experimentally, for instance, in the context of cold atoms [65-67].

In this work, the dynamics of the system are characterized by studying the time evolution of the entanglement between a contiguous subset of $N$ spins $A=\{1,2, \ldots N\}$ and the rest of the system; see Fig. 1. The entanglement is encoded in the density matrix of the system reduced to the subsystem $A$ defined as

$$
\rho_{A}(t)=\operatorname{tr}_{\mathcal{H}_{L-N}}\left[\left|\psi_{\boldsymbol{\theta}, \boldsymbol{\phi}}(t)\right\rangle\left\langle\psi_{\boldsymbol{\theta}, \boldsymbol{\phi}}(t)\right|\right],
$$

where $\mathcal{H}_{L-N}$ is the Hilbert space associated with the complement $A^{c}=\{N+1, \ldots, L\}$ of $A$. The entanglement content of $\rho_{A}(t)$ is quantified by the Rényi entropies $S_{A}^{(\alpha)}(t)$, also called entanglement entropies. These are a oneparameter family of functionals of $\rho_{A}(t)$ defined as follows: 


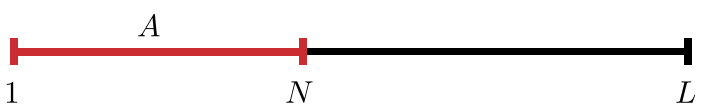

FIG. 1. The partition of the spin chain considered in the calculation of the entanglement.

$$
S_{A}^{(\alpha)}(t)=\frac{1}{1-\alpha} \log \operatorname{tr}\left[\left(\rho_{A}(t)\right)^{\alpha}\right], \quad \alpha>0 .
$$

A particularly important member of this family is the von Neumann entropy

$$
S_{A}^{(\mathrm{vN})}(t)=\lim _{\alpha \rightarrow 1} S_{A}^{(\alpha)}(t)=-\log \operatorname{tr}\left[\rho_{A}(t) \log \rho_{A}(t)\right],
$$

which is the most used measure of bipartite entanglement for pure states [3].

In summary, in this paper we study the time evolution generated by Eq. (1) of the Rényi entropies (15) for a system initialized in the states $\left|\psi_{\theta, \phi}\right\rangle$. As we see, our analytical results apply in the thermodynamic limit $L \rightarrow \infty$. We stress that, in contrast with what we did elsewhere [56], we do not introduce any averaging on the longitudinal magnetic fields. The thermodynamic limit is taken for fixed initial state and time-evolving Hamiltonian.

\section{OUTLINE OF THE RESULTS}

Our main result consists in finding two specific but physically interesting subclasses of the states (12) for which the time evolution of all Rényi entropies in the thermodynamic limit can be found exactly for any configuration of magnetic fields $\left\{h_{j}\right\}$ and subsystem size $N$. These special classes of states are defined as

$$
\begin{gathered}
\mathcal{T}=\left\{\left|\psi_{(\pi / 2) \mathbf{1}, \boldsymbol{\phi}}\right\rangle, \quad \phi_{j} \in[0,2 \pi]\right\}, \\
\mathcal{L}=\left\{\left|\psi_{\overline{\boldsymbol{\theta}}, \boldsymbol{\phi}}\right\rangle, \quad \bar{\theta}_{j} \in\{0, \pi\}\right\},
\end{gathered}
$$

where 1 denotes a vector of length $L$ with all entries equal to 1 [68]. We, respectively, name them "transverse separating states" and "longitudinal separating states," while we generically call "separating state" a state belonging to either $\mathcal{T}$ or $\mathcal{L}$. These states are called "transverse" and "longitudinal" because they are, respectively, eigenstates of the operators $\cos \phi_{j} \sigma_{j}^{x}+\sin \phi_{j} \sigma_{j}^{y}$ and $\sigma_{j}^{z}$ for all $j$ 's. Therefore, they can be thought of as configurations of a magnet where the spins lie on the $x-y$ plane ("transverse plane") or along the $z$ axis ("longitudinal axis"). The adjective "separating" refers to their key mathematical property, and it is thoroughly explained in Sec. V. We stress that the property of being separating is more restrictive that being just separable: All the states $\left|\psi_{\boldsymbol{\theta}, \boldsymbol{\phi}}\right\rangle$ are separable but only a subset of them are separating. Specific instances of separating states, which are most relevant from the experimental point of view, are the ground states of the two parts in the Floquet protocol. For example, when $J>0$ and $\left|h_{j}\right|<J$, the ground state of $H_{I}$ is $\left|\psi_{\pi \mathbf{1 0}}\right\rangle \in \mathcal{L}$, while when $b>0$, the ground state of $H_{K}$ is $\left|\psi_{(\pi / 2) \mathbf{1}, \pi \mathbf{1}}\right\rangle \in \mathcal{T}$.

To simplify the analysis of the results, it is useful to note that the time evolution of the states in $\mathcal{L}$ can be related to that of those in $\mathcal{T}$. This is easily seen by means of the following identity

$$
\left|\psi_{\overline{\boldsymbol{\theta}}, \boldsymbol{\phi}}(1)\right\rangle=U_{K I}[\boldsymbol{h}]\left|\psi_{\overline{\boldsymbol{\theta}}, \boldsymbol{\phi}}\right\rangle \simeq\left|\psi_{(\pi / 2) \mathbf{1}, \overline{\boldsymbol{\theta}}-(\pi / 2) \mathbf{1}}\right\rangle,
$$

where $\simeq$ denotes equality up to a global phase. This identity is proven by observing that, since the states in $\mathcal{L}$ are eigenstates of $\sigma_{j}^{z}$, they are also eigenstates of $H_{I}[\boldsymbol{h}]$. Therefore, the application of $e^{-i H_{I}[\boldsymbol{h}]}$ changes only $\left|\psi_{\overline{\boldsymbol{\theta}}, \boldsymbol{\phi}}\right\rangle$ by a global phase. Moreover, an explicit calculation shows that

$$
e^{-i H_{K}}\left|\psi_{\overline{\boldsymbol{\theta}}, \boldsymbol{\phi}}\right\rangle \simeq\left|\psi_{(\pi / 2) \mathbf{1}, \overline{\boldsymbol{\theta}}-(\pi / 2) \mathbf{1}}\right\rangle .
$$

Equation (19) means that the first time step of evolution for states in $\mathcal{L}$ keeps them in a separable form, and hence does not change the entanglement but turns them into states in $\mathcal{T}$. An immediate consequence of Eq. (19) is

$$
\left|\psi_{\overline{\boldsymbol{\theta}}, \boldsymbol{\phi}}(t)\right\rangle \simeq\left|\psi_{(\pi / 2) \mathbf{1}, \overline{\boldsymbol{\theta}}-(\pi / 2) \mathbf{1}}(t-1)\right\rangle, \quad t \geq 1 .
$$

Considering the entanglement entropy, we then have

$\left.S_{A}^{(\alpha)}(t)\right|_{\overline{\boldsymbol{\theta}}_{, \boldsymbol{\phi}}}=\left.S_{A}^{(\alpha)}[\max (t-1,0)]\right|_{(\pi / 2) \mathbf{1}, \overline{\boldsymbol{\theta}}-(\pi / 2) \mathbf{1}}, \quad t \geq 0$,

where we explicitly report the initial-state dependence and use $S_{A}^{(\alpha)}(0)=0$. By virtue of this simple argument, we can restrict our attention to the states in $\mathcal{T}$, and the time evolution of the entropy for the states in $\mathcal{L}$ is found using Eq. (22).

The time evolution of entanglement entropies from states in $\mathcal{T}$ (in the thermodynamic limit) can be explicitly determined by means of the "duality method" developed in Secs. IV-VI. The result reads as

$$
\lim _{L \rightarrow \infty} S_{A}^{(\alpha)}(t)=\min (2 t, N) \log 2
$$

This result is indisputably remarkable: When evolving from separating states, all entanglement entropies take the same universal form independent of the fields $h_{j}$ and details of the initial states. In particular, at fixed $N$, the entanglement entropies display a linear growth in time up to a nonanalytic saturation point where they become constant; see Fig. 2. The independence on $\alpha$ of the result means that the spectra of the reduced density matrices $\rho_{A}(t)$ are flat. In other 


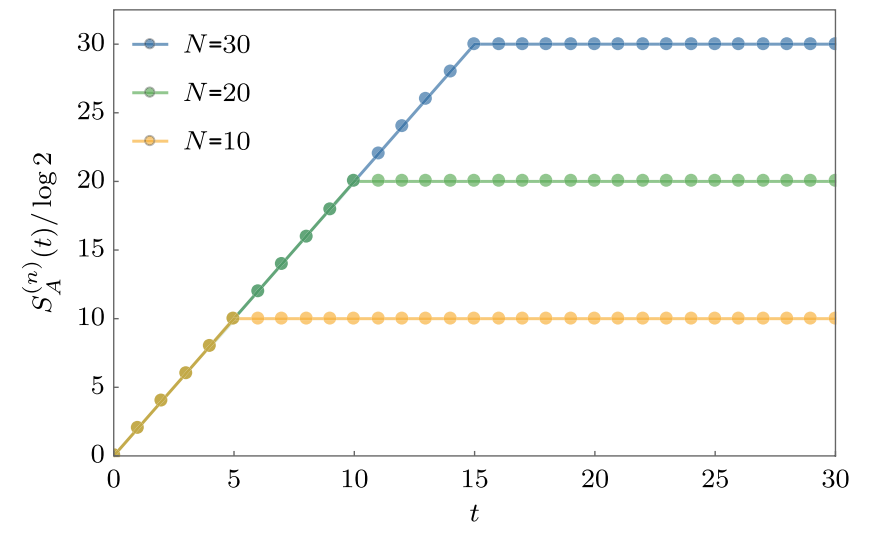

FIG. 2. Plot of the exact result of all Rényi entropies given by Eq. (23) for the $\theta=\pi / 2$ case. The expressions for $\theta=0$ are delayed by one period.

words, the reduced density matrices have exactly $2^{\min (2 t, N)}$ eigenvalues equal to $2^{-\min (2 t, N)}$ while the others vanish.

A form like Eq. (23) for the evolution of the entanglement entropies has been found in a number of different physical settings, both in closed and periodically driven systems. Examples range from conformal invariant systems [15,33], to nonintegrable closed systems [43], from random in time [50-52], to periodically driven [55] random unitary circuits. As opposed to all these cases, however, our result does not hold only at the leading order for large $t$ and $N$. It holds with no corrections for any $t$ and $N$. This gives further evidence for the special status of the self-dual kicked Ising model as a minimal solvable model for quantum chaos.

Another interesting feature of our result is that it saturates the "minimal cut" bound [34]. The bound states that, when evolving from a product state,

$$
S_{A}^{(\alpha)}(t) \leq \ell_{\min } \log q, \quad \forall \alpha,
$$

where $q$ is the dimension of the local Hilbert space ( 2 in our case), and $\ell_{\min }$ is the minimal number of links intersected by a cut separating the region $A$ from the rest of the system in a local quantum-circuit representation of $U_{K I}^{t}$. The fact that the bound is saturated means that entanglement spreads with the maximal possible speed allowed by the range of the Hamiltonian and the dimension of the local Hilbert space. This fact can be seen in a more physical way by noting that Eq. (23) implies that, for $t \leq N / 2$, at each time step two more spins of the block $A$ become maximally entangled with the rest of the system. Following Ref. [33], this phenomenon can be pictured as an entanglement wave propagating into the block $A$ from the two boundaries. The fact that our exact result (23) saturates the bound (24) also means that it agrees with the "minimal membrane" picture recently put forward in Ref. [50], where a coarse-grained version of the minimal cut has been proposed to describe the leading-order-in-time features of the entanglement dynamics in generic systems. Interestingly, however, our system also contains an integrable point, namely, $\boldsymbol{h}=\mathbf{0}$. At this point, our result agrees with the quasiparticle picture of Ref. [15], because in our case, all quasiparticles move at the same maximal speed $(|v|=1)$. We note that, at the integrable point, the result (23) has also been found in Ref. [69] for the evolution of the von Neumann entanglement from the separating state $\left|\psi_{(\pi / 2) \mathbf{1 , 0}}\right\rangle$.

If the initial state is not separating, the problem is not amenable to an analytical treatment. In Sec. VII, however, performing a thorough numerical analysis, we argue that the entanglement spreading from all the states (12) is still described by Eq. (23) at the leading order in time, provided that the system is away from the integrable point $\boldsymbol{h}=\mathbf{0}$. Note that this conjecture is physically very reasonable: Since the system is quantum ergodic, it is natural to expect the entanglement entropies to eventually become state independent. On the contrary, in the integrable case, our numerical results are consistent with

$$
\lim _{L \rightarrow \infty} S_{A}^{(\alpha)}(t)=\min (2 t, N) S_{\boldsymbol{\theta}, \boldsymbol{\phi}}^{(\alpha)}
$$

where $S_{\boldsymbol{\theta}, \boldsymbol{\phi}}^{(\alpha)} \leq \log 2$ is an initial-state-dependent constant. In the numerical analysis that leads to these results, a crucial role is played by a duality-based numerical approach (see Sec. VII) that allows us to treat the system in the infinite volume limit. Supplemented with some analytical information, it can reach $t=17$ Floquet periods of evolution even in cases where the entanglement grows at the maximal speed. Even if based on the "duality method," this approach does not rely on the special algebraic structure arising at the self-dual points (4), and it is applicable in the whole parameter space of the kicked Ising model.

Another marked difference between the integrable and nonintegrable case is observed when the system is confined in a finite volume $L$. Indeed, in this setting the integrable system displays finite-size-related recurrences when $t \sim L$, while these recurrences are absent (or at least negligible) in the nonintegrable case. These results, respectively, agree with the predictions of the quasiparticle and the minimal membrane pictures and are also consistent with the numerical analysis of the entanglement spreading in the kicked Ising model carried out in Ref. [44].

\section{DUALITY MAPPING FOR THE ENTANGLEMENT ENTROPIES}

In Ref. [70], the authors pointed out that the traces of integer powers of the Floquet operator (8) enjoy a useful space-time "duality symmetry," which can be demonstrated as follows. First, note that

$$
\operatorname{tr}\left[\left(U_{K I}[\boldsymbol{h}]\right)^{t}\right], \quad t \in \mathbb{N}
$$

can be represented as a partition function of a classical Ising model on a $t \times L$ lattice, where $U_{K I}[\boldsymbol{h}]$ acts as transfer 


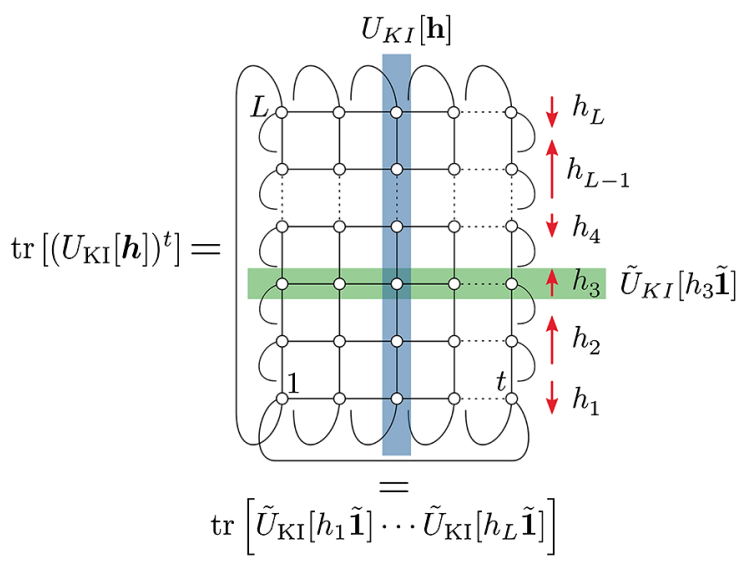

FIG. 3. Pictorial representation of the duality relation (27) fulfilled by the Floquet operator (8). Traces of powers of the Floquet operator correspond to the partition function of a classical Ising model on a $t \times L$ lattice. The column-to-column transfer matrix is given by $U_{K I}[\boldsymbol{h}]$, while the row-to-row transfer matrix between the $(j-1)$ th, and the $j$ th row is given by $\tilde{U}_{K I}\left[h_{j} \tilde{\mathbf{1}}\right]$. Note that self-duality condition implies that both transfer matrices are unitary.

matrix in time; see Fig. 3 for a pictorial representation and Appendix A for the explicit expression. Second, observe that, due to the short-range nature of the couplings in Eqs. (2) and (3), the same quantity can also be written in terms of a transfer matrix defined on a lattice of $t$ sites and propagating in space (see Appendix A). Namely, we have

$$
\operatorname{tr}\left[\left(U_{K I}[\boldsymbol{h}]\right)^{t}\right]=\operatorname{tr}\left[\tilde{U}_{K I}\left[h_{1} \tilde{\mathbf{1}}\right] \cdots \tilde{U}_{K I}\left[h_{L} \tilde{\mathbf{1}}\right]\right],
$$

where "tilded" bold symbols denote vectors of $t$ components, in particular,

$$
\tilde{\mathbf{1}}=\underbrace{(1, \ldots, 1)}_{t}
$$

has all entries equal to 1 , and $\tilde{U}_{K I}[\tilde{\boldsymbol{h}}]$ is the transfer matrix in space, also called the "dual" transfer matrix. It turns out that $\tilde{U}_{K I}[\tilde{\boldsymbol{h}}]$ has the same form as the Floquet operator (8) [with $L$ replaced by $t$ in Eqs. (2) and (3)] where the longitudinal magnetic field vector is given by $\tilde{\boldsymbol{h}}$, while Ising coupling $\tilde{J}$ and the transverse field $\tilde{b}$ are given by the following functions of $J$ and $b$ :

$$
\begin{aligned}
& \tilde{J}=-\frac{\pi}{4}-\frac{i}{2} \log \tan b, \\
& \tilde{b}=-\frac{\pi}{4}-\frac{i}{2} \log \tan J .
\end{aligned}
$$

Since $\tilde{J}$ and $\tilde{b}$ are generically complex, the transfer matrix $\tilde{U}_{K I}[\tilde{\boldsymbol{h}}]$ is generically not unitary. The dual couplings become real only when the model is at one of the selfdual points (4).

In Ref. [56], we showed that such a duality symmetry can be used to compute nontrivial observables, considering the example of the disorder-averaged spectral form factor. In that case, even if the quantity cannot be written in terms of a transfer matrix in time, it can still be written in terms of a transfer matrix in space. This allowed us to perform an analytical calculation. The unitarity of the matrix $\tilde{U}_{K I}[\tilde{\boldsymbol{h}}]$, however, proved itself to be a necessary requirement for the analytical approach to be feasible. This clarifies the special status of the self-dual points (4): They are the only points of the parameter space where this duality mapping leads to an analytic solution.

Here we develop a similar duality mapping for the calculation of the entanglement entropies, or, more precisely, of the traces of integer powers of the reduced density matrix $\rho_{A}(t)$. We see that also $\operatorname{tr}\left[\left(\rho_{A}(t)\right)^{n}\right]$ can be written as the trace of a power of an appropriate transfer matrix in time. In Secs. V and VI, we then show that, at the self-dual points and for the special initial states (17) and (18), such a trace can be analytically evaluated.

Considering $\operatorname{tr}\left[\left(\rho_{A}(t)\right)^{n}\right]$ and using the definitions (13) and (14), we find

$$
\begin{aligned}
& \operatorname{tr}\left[\left(\rho_{A}(t)\right)^{n}\right] \\
& =\sum_{\left\{\boldsymbol{a}_{i}\right\},\left\{\boldsymbol{b}_{i}\right\}}\left\langle\psi_{\boldsymbol{\theta}, \boldsymbol{\phi}}\left|\left(U_{K I}[\boldsymbol{h}]\right)^{-t}\right| \boldsymbol{a}_{1}, \boldsymbol{b}_{2}\right\rangle\left\langle\boldsymbol{a}_{1}, \boldsymbol{b}_{1}\left|\left(U_{K I}[\boldsymbol{h}]\right)^{t}\right| \psi_{\boldsymbol{\theta}, \boldsymbol{\phi}}\right\rangle \\
& \quad \times\left\langle\psi_{\boldsymbol{\theta}, \boldsymbol{\phi}}\left|\left(U_{K I}[\boldsymbol{h}]\right)^{-t}\right| \boldsymbol{a}_{2}, \boldsymbol{b}_{3}\right\rangle\left\langle\boldsymbol{a}_{2}, \boldsymbol{b}_{2}\left|\left(U_{K I}[\boldsymbol{h}]\right)^{t}\right| \psi_{\boldsymbol{\theta}, \boldsymbol{\phi}}\right\rangle \\
& \vdots \\
& \quad \times\left\langle\psi_{\boldsymbol{\theta}, \boldsymbol{\phi}}\left|\left(U_{K I}[\boldsymbol{h}]\right)^{-t}\right| \boldsymbol{a}_{n}, \boldsymbol{b}_{1}\right\rangle\left\langle\boldsymbol{a}_{n}, \boldsymbol{b}_{n}\left|\left(U_{K I}[\boldsymbol{h}]\right)^{t}\right| \psi_{\boldsymbol{\theta}, \boldsymbol{\phi}}\right\rangle,
\end{aligned}
$$

where $\left|\boldsymbol{a}_{i}, \boldsymbol{b}_{j}\right\rangle=\left|\boldsymbol{a}_{i}\right\rangle \otimes\left|\boldsymbol{b}_{j}\right\rangle,\left|\boldsymbol{a}_{i}\right\rangle \in \mathcal{B}_{N},\left|\boldsymbol{b}_{i}\right\rangle \in \mathcal{B}_{L-N}$, for $i, j \in\{1,2, \ldots, n\}$. Here we denote by $\mathcal{B}_{j}$ the computational basis of

$$
\mathcal{H}_{j}=\left(\mathbb{C}^{2}\right)^{\otimes j}
$$

An explicit expression of $\mathcal{B}_{j}$ is obtained replacing $L$ by $j$ in the expression (7).

Equation (31) allows one to interpret the trace of the $n$th power of the reduced density matrix as the partition function of a classical statistical mechanical model on a multisheeted two-dimensional lattice; see Fig. 4 for a pictorial representation in the case $n=3$. To see it more explicitly, we consider a single "building block"

$$
\left\langle\boldsymbol{a}, \boldsymbol{b}\left|\left(U_{K I}[\boldsymbol{h}]\right)^{t}\right| \psi_{\boldsymbol{\theta}, \boldsymbol{\phi}}\right\rangle,
$$

and show that it is equivalent to the partition function of a classical Ising model (with complex weights) on a $t \times L$ lattice with periodic boundary conditions in space and fixed boundary conditions in time. This is seen in two steps. 


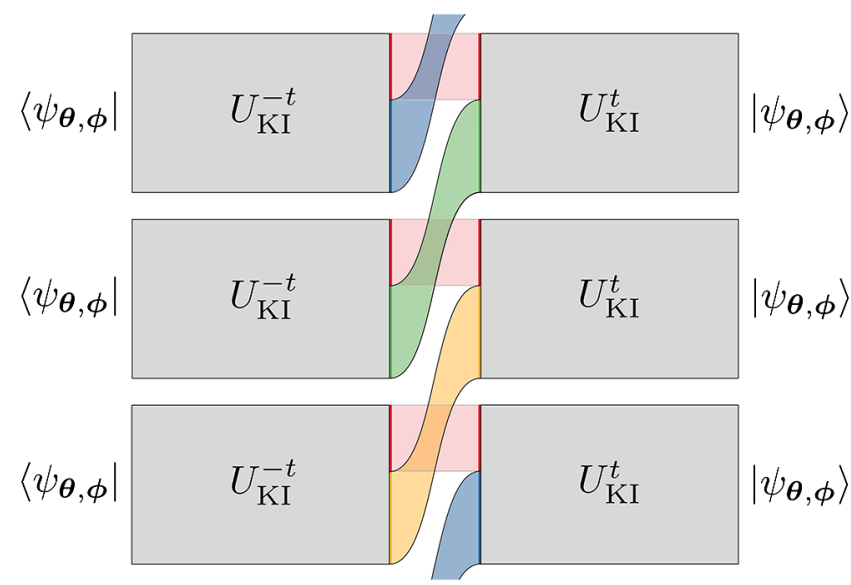

FIG. 4. Schematic representation of $\operatorname{tr}\left[\left(\rho_{A}(t)\right)^{3}\right]$ according to the expression (31). The six different cylinders corresponding to the partition functions (38) are schematically represented as rectangles. The spin subchains $A$ and $A^{c}$ connected with the colored belts share identical spin configurations.

First, we insert $t$ resolutions of the identity operator in the basis (7) into Eq. (33), obtaining

$$
\begin{aligned}
\left\langle\boldsymbol{a}, \boldsymbol{b}\left|\left(U_{K I}[\boldsymbol{h}]\right)^{t}\right| \psi_{\boldsymbol{\theta}, \boldsymbol{\phi}}\right\rangle= & \sum_{\left\{\boldsymbol{s}_{\tau}\right\}} \prod_{\tau=1}^{t-1}\left\langle\boldsymbol{s}_{\tau+1}\left|U_{K I}[\boldsymbol{h}]\right| \boldsymbol{s}_{\tau}\right\rangle \\
& \times\left\langle\boldsymbol{a}, \boldsymbol{b}\left|U_{K I}[\boldsymbol{h}]\right| \boldsymbol{s}_{t}\right\rangle\left\langle\boldsymbol{s}_{1} \mid \psi_{\boldsymbol{\theta}, \boldsymbol{\phi}}\right\rangle .
\end{aligned}
$$

Then we evaluate the matrix elements

$$
\left\langle\boldsymbol{s} \mid \psi_{\boldsymbol{\theta}, \boldsymbol{\phi}}\right\rangle=\prod_{j=1}^{L}\left[\cos \left(\theta_{j} / 2\right) \delta_{s_{j}, 1}+\sin \left(\theta_{j} / 2\right) e^{i \phi_{j}} \delta_{s_{j},-1}\right]
$$

and

$$
\begin{aligned}
\left\langle\boldsymbol{s}\left|U_{K I}[\boldsymbol{h}]\right| \boldsymbol{r}\right\rangle= & \left(\frac{i}{2}\right)^{(L / 2)} \exp \left[-\frac{i \pi}{4} \sum_{j=1}^{L} s_{j} r_{j}\right] \\
& \times \exp \left[-\frac{i \pi}{4} \sum_{j=1}^{L} r_{j} r_{j+1}-i \sum_{j=1}^{L} h_{j} r_{j}\right] .
\end{aligned}
$$

Here, to find the last equation, we set $r_{L+1}=r_{1}$ and we use the identity

$$
\left\langle s\left|e^{i(\pi / 4) \sigma^{x}}\right| r\right\rangle=\sqrt{\frac{i}{2}} \exp \left[-i \frac{\pi}{4} s r\right], \quad s, r \in\{ \pm 1\}
$$

to treat the "kick" part of the Floquet operator $U_{K I}[\boldsymbol{h}]$. Putting them all together, we have

$$
\begin{aligned}
& \left\langle\boldsymbol{a}, \boldsymbol{b}\left|\left(U_{K I}[\boldsymbol{h}]\right)^{t}\right| \psi_{\boldsymbol{\theta}, \boldsymbol{\phi}}\right\rangle \\
& =\left(\frac{i}{2}\right)^{(t L) / 2} \sum_{\left\{s_{\tau, j}\right\}} \exp \left[-\frac{i \pi}{4} \sum_{\tau=1}^{t} \sum_{j=1}^{L} s_{\tau, j} s_{\tau, j+1}-\frac{i \pi}{4} \sum_{\tau=1}^{t-1} \sum_{j=1}^{L} s_{\tau, j} s_{\tau+1, j}-i \sum_{\tau=1}^{t} \sum_{j=1}^{L} h_{j} s_{\tau, j}\right] \\
& \quad \times \exp \left[-\frac{i \pi}{4} \sum_{j=1}^{N} s_{t, j} a_{j}-\frac{i \pi}{4} \sum_{j=N+1}^{L} s_{t, j} b_{j-N}\right] \prod_{j=1}^{L}\left[\cos \left(\theta_{j} / 2\right) \delta_{s_{1, j}, 1}+\sin \left(\theta_{j} / 2\right) e^{i \phi_{j}} \delta_{s_{1, j},-1}\right],
\end{aligned}
$$

which, as promised, is the partition function of the classical Ising model on a two-dimensional cylinder.

Representing in this way each of the $2 n$ building blocks in Eq. (31) and summing over $\left\{\boldsymbol{a}_{j}, \boldsymbol{b}_{j}\right\}$, one connects together the $2 n$ different cylinders obtaining the announced multisheeted lattice. Explicitly, we have

$$
\begin{aligned}
\operatorname{tr}\left\{\left[\rho_{A}(t)\right]^{n}\right\}= & \frac{1}{2^{n L t}} \sum_{\left\{s_{\nu, \tau, j}\right\}} \exp \left[-i \sum_{j=1}^{L} \sum_{\nu=1}^{2 n} \operatorname{sgn}(n-\nu)\left(\sum_{\tau=1}^{t}\left(\frac{\pi}{4} s_{\nu, \tau, j} s_{\nu, \tau, j+1}+h_{j} s_{\nu, \tau, j}\right)+\sum_{\tau=1}^{t-1} \frac{\pi}{4} s_{\nu, \tau, j} s_{\nu, \tau+1, j}\right)\right] \\
& \times \prod_{\nu=1}^{n}\left\{\prod_{j=1}^{N}\left(1+s_{\nu, t, j} s_{\nu+n, t, j}\right) \prod_{j=N+1}^{L}\left(1+s_{\nu, t, j} s_{n+1+\bmod (\nu-2, n), t, j}\right)\right\} \\
& \times \prod_{\nu=1}^{2 n} \prod_{j=1}^{L}\left(\cos \left(\theta_{j} / 2\right) \delta_{s_{\nu, 1, j}, 1}+\sin \left(\theta_{j} / 2\right) e^{i \phi_{j} \operatorname{sgn}(n-\nu)} \delta_{s_{\nu, 1, j},-1}\right),
\end{aligned}
$$

where $\operatorname{sgn}(x)$ is the sign function [we adopt the convention $\operatorname{sgn}(0)=1], \bmod (m, n)=m \bmod n$ is the $\bmod$ function, and we introduce a new index $\nu \in\{1,2, \ldots, 2 n\}$ such that strings $\boldsymbol{s}_{\nu, \tau}$ with $\nu \leq n$ belong to the terms in Eq. (31) with forward time evolution, while those with $\nu>n$ belong to the terms in Eq. (31) with backward time evolution. 
The second line of Eq. (39) is obtained by explicitly summing over $\left\{\boldsymbol{a}_{i}\right\}$ and $\left\{\boldsymbol{b}_{i}\right\}$ with the help of the identity

$$
\sum_{a \in\{ \pm 1\}} \exp \left[-i \frac{\pi}{4} a(s-r)\right]=1+s r, \quad s, r \in\{ \pm 1\} .
$$

We see that this line forces the configurations of spins in the subchains $A$ and $A^{c}$ on the edges of different cylinders to be the same. These "frozen" configurations are represented by colored strips in Fig. 4.

To proceed, it is useful to introduce the tensor product space $\mathcal{H}_{t}^{\otimes 2 n}$ composed of $2 n$ copies of $\mathcal{H}_{t}$, which is the space where the dual Floquet operator $\tilde{U}_{K I}[\tilde{\boldsymbol{h}}]$ acts. More formally,

$$
\mathcal{H}_{t}^{\otimes 2 n}=\overbrace{\mathcal{H}_{t} \otimes \cdots \otimes \mathcal{H}_{t}}^{2 n} \cong \mathcal{H}_{2 n t} .
$$

Then, we define the operators $\mathbb{T}_{\theta, \phi}[h]$ and $\mathbb{R}_{\theta, \phi}[h]$ on $\mathcal{H}_{t}^{\otimes 2 n}$ through their matrix elements in the computational basis

$$
\begin{aligned}
\left\langle\left\{s_{\nu, \tau}\right\}\left|\mathbb{T}_{\theta, \phi}[h]\right|\left\{r_{\nu, \tau}\right\}\right\rangle= & \frac{1}{2^{(t-1) n}} \exp \left[-i \sum_{\nu=1}^{2 n} \operatorname{sgn}(n-\nu)\left(\sum_{\tau=1}^{t}\left(\frac{\pi}{4} s_{\nu, \tau} r_{\nu, \tau}+h_{j} s_{\nu, \tau}\right)+\sum_{\tau=1}^{t-1} \frac{\pi}{4} s_{\nu, \tau} s_{\nu, \tau+1}\right)\right] \\
& \times \prod_{\nu=1}^{n}\left(\frac{1+s_{\nu, t} s_{\nu+n, t}}{2}\right) \prod_{\nu=1}^{2 n}\left(\cos (\theta / 2) \delta_{s_{\nu, 1}, 1}+\sin (\theta / 2) e^{i \phi \operatorname{sgn}(n-\nu)} \delta_{s_{\nu, 1},-1}\right)
\end{aligned}
$$

and

$$
\begin{aligned}
\left\langle\left\{s_{\nu, \tau}\right\}\left|\mathbb{R}_{\theta, \phi}[h]\right|\left\{r_{\nu, \tau}\right\}\right\rangle= & \frac{1}{2^{(t-1) n}} \exp \left[-i \sum_{\nu=1}^{2 n} \operatorname{sgn}(n-\nu)\left(\sum_{\tau=1}^{t}\left(\frac{\pi}{4} s_{\nu, \tau} r_{\nu, \tau}+h_{j} s_{\nu, \tau}\right)+\sum_{\tau=1}^{t-1} \frac{\pi}{4} s_{\nu, \tau} s_{\nu, \tau+1}\right)\right] \\
& \times \prod_{\nu=1}^{n}\left(\frac{1+s_{\nu, t} s_{n+1+\bmod (\nu-2, n), t}}{2}\right) \prod_{\nu=1}^{2 n}\left(\cos (\theta / 2) \delta_{s_{\nu, 1}, 1}+\sin (\theta / 2) e^{i \phi \operatorname{sgn}(n-\nu)} \delta_{s_{\nu, 1},-1}\right),
\end{aligned}
$$

where the first subscript labels spin variables in the different copies of $\mathcal{H}_{t}$ composing $\mathcal{H}_{t}^{\otimes 2 n}$.

Using the above matrix elements, it is immediate to see that the expression (39) can directly be rewritten as a trace (on $\mathcal{H}_{t}^{\otimes 2 n}$ ) of products of $\mathbb{T}_{\theta, \phi}[h]$ and $\mathbb{R}_{\theta, \phi}[h]$, namely,

$\operatorname{tr}\left[\left(\rho_{A}(t)\right)^{n}\right]=\operatorname{tr}\left[\left(\prod_{j=1}^{N} \mathbb{T}_{\theta_{j}, \phi_{j}}\left[h_{j}\right]\right)\left(\prod_{j=N+1}^{L} \mathbb{R}_{\theta_{j}, \phi_{j}}\left[h_{j}\right]\right)\right]$,

where we define on ordered product of noncommuting operators $\left\{\mathbb{O}_{j}\right\}$ as

$$
\prod_{j=a}^{b} \mathbb{O}_{j}= \begin{cases}\mathbb{O}_{a}, \ldots, \mathbb{O}_{b} & \text { if } a \leq b, \\ \mathbb{1} & \text { if } a>b .\end{cases}
$$

The rewriting achieved by Eq. (44) is pictorially represented in Fig. 5, again in the case $n=3$.

In an upcoming analysis, it will be useful to think of $\mathcal{H}_{t}^{\otimes 2 n}$ as a tensor product of two copies of $\mathcal{H}_{n t}$, grouping together the first and the last $n$ copies of $\mathcal{H}_{t}$; see Fig. 6 . Namely, we write each element of the basis of $\mathcal{H}_{t}^{\otimes 2 n}$ in the following way:

$$
\left|\left\{s_{a, \tau}\right\}_{1 \leq \tau \leq t}^{1 \leq a \leq 2 n}\right\rangle=\left|\left\{s_{a, \tau}\right\}_{1 \leq \tau \leq t}^{1 \leq a \leq n}\right\rangle \otimes\left|\left\{s_{a, \tau}\right\}_{1 \leq \tau \leq t}^{n<a \leq 2 n}\right\rangle .
$$

We call these two copies of $\mathcal{H}_{n t}$ the "positive-time" and "negative-time" spaces, respectively, as the components of $\mathbb{T}_{\theta, \phi}[h]$ and $\mathbb{R}_{\theta, \phi}[h]$ acting on those spaces come from terms in Eq. (31) propagating forward and backward in time, respectively.

It is useful to note that $\mathbb{T}_{\theta, \phi}[h]$ and $\mathbb{R}_{\theta, \phi}[h]$ are the same up to a cyclic permutation of the copies of $\mathcal{H}_{t}$ composing the negative-time space (i.e., a cyclic permutation of the second row of Fig. 6), namely,

$$
\mathbb{R}_{\theta, \phi}[h]=\mathbb{P} \mathbb{T}_{\theta, \phi}[h] \mathbb{P}^{\dagger},
$$

where we define 


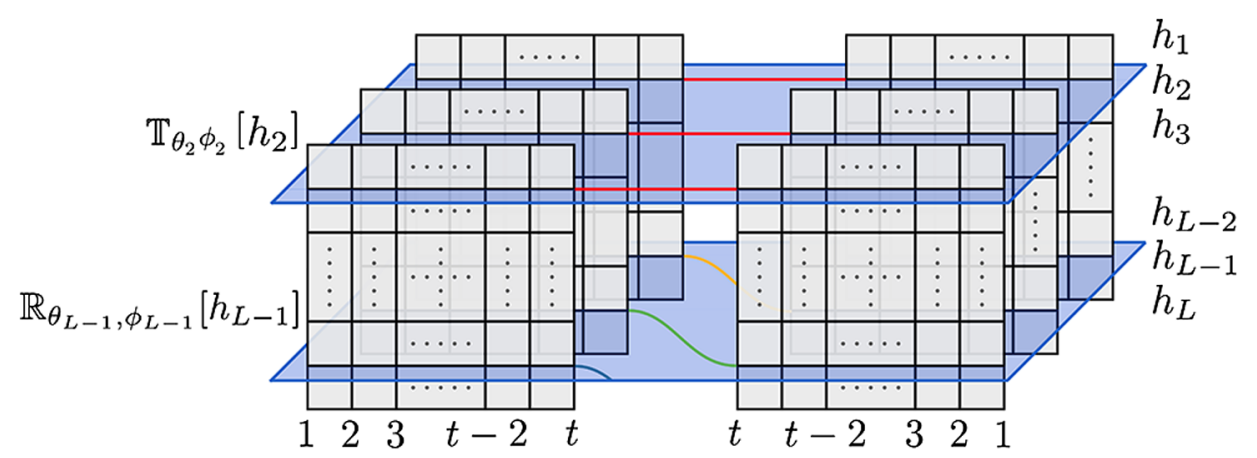

FIG. 5. Schematic depiction of $\operatorname{tr}\left[\left(\rho_{A}(t)\right)^{3}\right]$ written according to Eq. (44). Positive and negative time sheets are, respectively, on the left and on the right. Vertices connected by the colored lines are coupled by the transfer matrices, in analogy with Fig. 4. Blue-shaded horizontal planes denote the spatial transfer matrices, specifically, the operator $\mathbb{T}_{\theta, \phi}[h]$ for the physical sites corresponding to the block $A$ of $N$ spins and the operator $\mathbb{R}_{\theta, \phi}[h]=\mathbb{P} \mathbb{T}_{\theta, \phi}[h] \mathbb{P}^{\dagger}$ for the sites corresponding to $L-N$ spins in $A^{c}$.

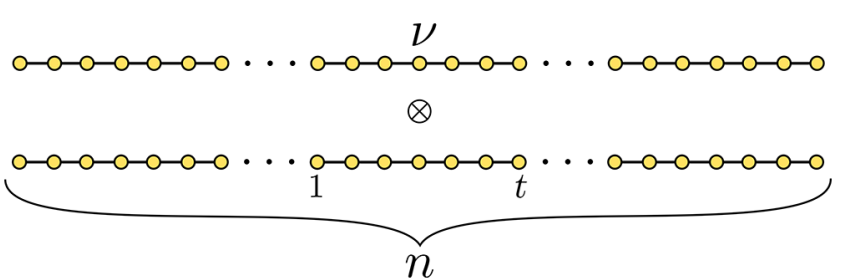

FIG. 6. Pictorial representation of the arrangement of the dual quantum spin degrees of freedom adopted in the tensor product space $\mathcal{H}_{t}^{\otimes 2 n}$.

$$
\mathbb{P}=\mathbb{1} \otimes \prod_{\nu=1}^{n} \prod_{\tau=1}^{t} P_{(\nu, \tau),(\nu-1, \tau)} .
$$

Here, $P_{(\nu, \tau),(\nu-1, \tau)}$ is an elementary transposition

$$
P_{(\nu, \tau),\left(\nu^{\prime}, \tau^{\prime}\right)}=\frac{1}{2} \mathbb{1}+\frac{1}{2} \sum_{a \in\{x, y, z\}} \sigma_{\nu, \tau}^{a} \sigma_{\nu^{\prime}, \tau^{\prime}}^{a}
$$

with $\nu, \nu^{\prime} \in\{1, \ldots, n\}$ and $\tau, \tau^{\prime} \in\{1, \ldots, t\}$. The matrix $\sigma_{\nu, \tau}^{a}$ acts as the Pauli matrix $\sigma^{a}, a \in\{x, y, z\}$, at the site $\tau=1, \ldots, t$ of the $\nu$ th copy of $\mathcal{H}_{t}$ in $\mathcal{H}_{n t}$, i.e.,

$$
\left[\sigma_{\nu, \tau}^{a}, \sigma_{\nu^{\prime}, \tau^{\prime}}^{b}\right]=2 i \delta_{\nu, \nu^{\prime}} \delta_{\tau, \tau^{\prime}} \varepsilon^{a b c} \sigma_{\nu, \tau}^{c}, \quad \sigma_{0, \tau}^{a} \equiv \sigma_{n, \tau}^{a} .
$$

Note that the property $P_{(\nu, \tau),(\mu, \sigma)}=P_{(\nu, \tau),(\mu, \sigma)}^{-1}$ implies $\mathbb{P}^{\dagger}=\mathbb{P}^{-1}$.

Writing Eq. (42) in matrix form, we have that the transfer matrix is a simple tensor product of single-copy transfer matrices

$$
\mathbb{T}_{\theta, \phi}[h]=\prod_{\nu=1}^{n} \mathbb{T}_{\theta, \phi}^{(\nu)}[h] .
$$

The matrix $\mathbb{T}_{\theta, \phi}^{(\nu)}[h]$ acts nontrivially only on the $\nu$ th copy of $\mathcal{H}_{t}$ in both the positive-time and negative-time spaces ( $\nu$ th column of Fig. 6), and it is explicitly written as

$$
\mathbb{T}_{\theta, \phi}^{(\nu)}[h]=\mathbb{B}_{\nu, 1}^{z}[\theta] \cdot \mathbb{G}_{\nu, t}^{z} \cdot \mathbb{U}_{\phi}^{(\nu)}[h],
$$

where we introduce the Hermitian matrix $\mathbb{B}_{\nu, \tau}^{a}[\theta]$, the projector $\mathbb{G}_{\nu, \tau}^{a}$, and the unitary matrix $\mathbb{U}_{\phi}^{(\nu)}[h]$ defined as follows:

$$
\begin{gathered}
\mathbb{B}_{\nu, \tau}^{a}[\theta] \equiv 2\left[\cos (\theta / 2) P_{\nu, \tau}^{a,+}+\sin (\theta / 2) P_{\nu, \tau}^{a,-}\right]^{\otimes 2}, \\
\mathbb{G}_{\nu, \tau}^{a} \equiv \frac{1}{2}\left(\mathbb{1}+\sigma_{\nu, \tau}^{a} \otimes \sigma_{\nu, \tau}^{a}\right), \\
\mathbb{U}_{\phi}^{(\nu)}[h] \equiv U_{\nu, \phi} e^{-i h M_{\nu}^{z}} e^{i(\pi / 4) M_{\nu}^{x}} \otimes U_{\nu, \phi}^{*} e^{i h M_{\nu}^{z}} e^{-i(\pi / 4) M_{\nu}^{x},}
\end{gathered}
$$

and finally,

$$
\begin{gathered}
U_{\nu, \phi} \equiv \exp \left[-\frac{i \pi}{4} \sum_{\tau=1}^{t-1} \sigma_{\nu, \tau}^{z} \sigma_{\nu, \tau+1}^{z}-i \frac{\phi}{2} \sigma_{\nu, 1}^{z}\right], \\
M_{\nu}^{a} \equiv \sum_{\tau=1}^{t} \sigma_{\nu, \tau}^{a}, \\
P_{\nu, \tau}^{a, \pm} \equiv \frac{1}{2}\left(\mathbb{1} \pm \sigma_{\nu, \tau}^{a}\right) .
\end{gathered}
$$

Note that since

$$
\left[\mathbb{T}_{\theta, \phi}^{(\nu)}[h], \mathbb{T}_{\theta, \phi}^{(\mu)}[h]\right]=0, \quad \mu, \nu \in\{1, \ldots, n\},
$$

the order in the product (51) is irrelevant.

Putting everything together, we have

$$
\begin{aligned}
S_{A}^{(n)}(t)= & \frac{1}{1-n} \log \operatorname{tr}\left[\left(\prod_{j=1}^{N} \mathbb{T}_{\theta_{j}, \phi_{j}}\left[h_{j}\right]\right) \mathbb{P}\right. \\
& \left.\times\left(\prod_{j=N+1}^{L} \mathbb{T}_{\theta_{j}, \phi_{j}}\left[h_{j}\right]\right) \mathbb{P}^{\dagger}\right] .
\end{aligned}
$$

This equation accomplishes the duality mapping of the entanglement entropies: We write the entanglement entropies in terms of the trace of products of an appropriate transfer matrix in space. 
Before continuing with the evaluation of Eq. (60), two comments are in order. First, we note that the mapping described can be performed also when $J$ and $b$ in Eqs. (2) and (3) do not fulfill the self-duality condition (4). For generic $J$ and $b$, we obtain that the entropy is still given by Eq. (60), but the transfer matrix $\mathbb{T}_{\theta, \phi}[h]$ is modified in two ways. (i) The matrix $\mathbb{U}_{\phi}^{(\nu)}[h]$ is not unitary anymore. The Ising coupling in Eq. (56) replaced by $\tilde{J}$ [cf. Eq. (29)] and the transverse fields in Eq. (55) (the coefficients of $i M_{\nu}^{x}$ in the positive and negative time copy) are, respectively, replaced by $\tilde{b}$ and $\tilde{b}^{*}$ [cf. Eq. (30)]. (ii) The projector $\mathbb{G}_{\nu, t}^{z}$ in Eq. (60) is replaced by

$$
\cos \left[b\left(\sigma_{\nu, t}^{z} \otimes \mathbb{1}-\mathbb{1} \otimes \sigma_{\nu, t}^{z}\right)\right] .
$$

As we see in the next section, these changes are enough to hinder the analytical evaluation of Eq. (60); however, the duality approach can still be useful for perturbative calculations or numerical approaches (see Sec. VII).

We also observe that when the initial state is in the class $\mathcal{L}$ [cf. Eq. (18)], namely, when

$$
\theta_{j}=\bar{\theta}_{j} \in\{0, \pi\}, \quad j \in\{1,2, \ldots, L\},
$$

the expression (60) can be further simplified by effectively reducing the dimension of the space where the trace acts. This is explicitly shown in Appendix B. The final result is again of the form (60) with the replacement

$$
\begin{aligned}
\mathbb{T}_{\bar{\theta}_{j}, \phi_{j}}\left[h_{j}\right] & \mapsto \overline{\mathbb{T}}_{\pi / 2, \bar{\theta}_{j}-\pi / 2}\left[h_{j}\right], \\
\mathbb{P} & \mapsto \overline{\mathbb{P}} .
\end{aligned}
$$

Here the barred operators have exactly the same form as the nonbarred ones [respectively, Eqs. (51) and (48)] but act on $\mathcal{H}_{t-1}^{\otimes 2 n}$ instead of $\mathcal{H}_{t}^{\otimes 2 n}$. Note that this is nothing but a restatement of property (22).

\section{SEPARATING STATES}

Our goal is to use Eq. (60) to determine $S_{A}^{(n)}(t)$ in the thermodynamic limit. To do that, however, we need some information on the Jordan normal form of the matrix $\mathbb{T}_{\theta, \phi}[h]$. Indeed, since the matrix is not normal, it is not guaranteed to be (and it is generically not) diagonalizable.

As we prove in Appendix C, the forms (51) and (52) of the transfer matrix have some simple but useful consequences on its Jordan normal form. Specifically, we have

Property 1: The following facts hold

(i) $\left|\lambda_{j}\right| \leq \lambda_{\max } \equiv(1+|\cos \theta|)^{n}, \forall \lambda_{j} \in \operatorname{Spec}\left[\mathbb{T}_{\theta, \phi}[h]\right]$.

(ii) If an eigenvalue $\lambda$ of $\mathbb{T}_{\theta, \phi}[h]$ fulfills $|\lambda|=\lambda_{\max }$, then

(a) $\lambda$ has trivial Jordan blocks (its geometric and algebraic multiplicities coincide). (b) the associated left eigenvector $\langle A|$ satisfies

$$
\begin{gathered}
\langle A| \prod_{\nu=1}^{n} \mathbb{B}_{\nu, 1}^{z}[\theta]=\lambda_{\max }\langle A|, \\
\langle A| \prod_{\nu=1}^{n} \mathbb{G}_{\nu, t}^{z}=\langle A|, \\
\langle A| \prod_{\nu=1}^{n} \mathbb{U}_{\phi}^{(\nu)}[h]=e^{i \alpha}\langle A|, \quad \alpha \in \mathbb{R},
\end{gathered}
$$

where $\operatorname{Spec}[A]$ denotes the spectrum of the matrix $A$.

Property 1 introduces the crucial simplification of this work. If the maximal eigenvalues of $\mathbb{T}_{\theta, \phi}[h]$ saturate the bounds at point (i), the problem of finding the maximal eigenvalues of the transfer matrix is separated into three much easier ones consisting of finding eigenvalues and eigenvectors of simple Hermitian and unitary matrices.

The bound at point (i), however, cannot be always saturated. To see this, let us consider some constraints on the structure of the matrix $\mathbb{T}_{\theta, \phi}[h]$ coming from the identity (44). These are most easily found by considering the translational-invariant case

$$
h_{j}=h, \quad \theta_{j}=\theta, \quad \phi_{j}=\phi, \quad \forall j .
$$

Setting $N=0$ in Eq. (44), we have

$$
\operatorname{tr}\left[\left(\mathbb{T}_{\theta, \phi}[h]\right)^{L}\right]=\operatorname{tr}\left[(\rho(t))^{n}\right]=1, \quad \forall L, n,
$$

where in the second step we use that the state (13) is pure. This relation implies that the eigenvalues of $\mathbb{T}_{\theta, \phi}[h]$ are all 0 but one, which is equal to 1 . Moreover, the Jordan block corresponding to the eigenvalue 1 is one dimensional, while the eigenvalue 0 might have (and does have) a highly nontrivial Jordan structure. More formally,

(C1) $\operatorname{Spec}\left[\mathbb{T}_{\theta, \phi}[h]\right]=\{0,1\}$.

(C2) The geometric multiplicity

of the eigenvalue 1 is 1 .

From the conditions (69), it follows that the bound at point (i) of Property 1 can be saturated only when $\lambda_{\max }=1$. Note that the cases for which $\lambda_{\max }=1$ include $\theta=\pi / 2$, but also $\theta=0, \pi$. Indeed, in the latter case the matrix $\mathbb{T}_{\theta, \phi}[h]$ can be replaced by $\overline{\mathbb{T}}_{\pi / 2, \pi / 2-\theta}[h]$ [see Eq. (63) and Appendix B]. In other words, the requirement $\lambda_{\max }=1$ selects the two classes of states $\mathcal{T}$ and $\mathcal{L}$ introduced in Sec. III. This clarifies the meaning of their name. We call them "separating" states because if the initial state is one of them, the problem of finding the maximal eigenvalues of the transfer matrix (and the corresponding eigenvectors) can be separated. In the upcoming section, we explicitly solve the separated problem (64)-(66) for $\lambda_{\max }=1$, and, incidentally, we also verify that it has no solution for $\lambda_{\max } \neq 1$. 
Finally, we note that away from the self-dual points (4), the conditions (69) still hold and a property similar to Property 1 is still valid. In that case, however, the bound can never be saturated and no separation can be performed. This makes the problem analytically intractable, at least in an exact fashion.

\section{ENTANGLEMENT SPREADING FROM SEPARATING STATES}

Here we explicitly solve the entanglement evolution from separating states. In particular, in Sec. VI A we solve the separated problem (64)-(66) for $\lambda_{\max }=1$, and in Sec. VI B we evaluate Eq. (60). To be concrete, we focus on the initial states in the class $\mathcal{T}$, and the result for the states in the class $\mathcal{L}$ is obtained using Eq. (22).

\section{A. Maximal eigenvalues of the transfer matrix}

Our strategy is to determine the maximal eigenvalues of $\mathbb{T}_{\pi / 2, \phi}[h]$ and the associated eigenvectors by searching for all the vectors fulfilling Eqs. (64)-(66) with $\lambda_{\max }=1$. To simplify our analysis, we make two observations. First, we note that

$$
\mathbb{B}_{\nu, 1}^{z}[\pi / 2]=\mathbb{1} \otimes \mathbb{1},
$$

so that Eq. (64) becomes trivial. Second, we note that all $\mathbb{G}_{\nu, t}^{z}$ and $\mathbb{U}_{\phi}^{(\nu)}$ commute for different $\nu$ 's, so we can look for simultaneous eigenvectors. The problem is then reduced to finding all vectors $\langle A|$ fulfilling

$$
\begin{gathered}
\langle A| \mathbb{G}_{\nu, t}^{z}=\langle A|, \\
\langle A| \mathbb{U}_{\phi}^{(\nu)}=\langle A| e^{i \alpha_{\nu}}, \quad \alpha_{\nu} \in \mathbb{R}, \quad \forall \nu \in\{1, \ldots, n\} .
\end{gathered}
$$

To solve these equations, it is convenient to introduce the following one-to-one vector-to-operator mapping (cf. Ref. [56]) $\langle A| \leftrightarrow A$,

$$
\langle A|=\sum_{k, m}\langle m|A| k\rangle\langle k| \otimes\left\langle\left. m\right|^{*},\right.
$$

where $\{\langle k|\}$ is a basis of $\mathcal{H}_{n t}$, and $(\cdot)^{*}$ denotes complex conjugation in the computational basis $\mathcal{B}_{n t}$, such that

$$
\left\langle\left. k\right|^{*} O^{*} \mid m\right\rangle^{*}=\langle k|O| m\rangle^{*}
$$

for any operator $O$. Using the mapping (73), Eqs. (71) and (72) are directly rewritten in operatorial form as follows:

$$
\begin{gathered}
\sigma_{\nu, t}^{z} A=A \sigma_{\nu, t}^{z}, \\
U_{\nu, \phi} e^{-i h M_{\nu}^{z}} e^{i(\pi / 4) M_{\nu}^{x}} A=e^{i \alpha_{\nu}} A U_{\nu, \phi} e^{-i h M_{\nu}^{z}} e^{i(\pi / 4) M_{\nu}^{x},}
\end{gathered}
$$

for some $\alpha_{\nu} \in \mathbb{R}$ and all $\nu \in\{1, \ldots, n\}$. In this formulation, our goal is to find all independent linear operators $A$ over
$\mathcal{H}_{n t}$ solving the commutation relations (75) and (76). As we show in Appendix D, these commutation relations are equivalent to

$$
A \sigma_{\nu, \tau}^{a}=\sigma_{\nu, \tau}^{a} A
$$

for all $a \in\{x, y, z\}, \tau \in\{1, \ldots, t\}, \nu \in\{1, \ldots, n\}$. Namely, they are equivalent to requiring that $A$ commutes with the entire algebra of observables in $\mathcal{H}_{n t}$. Since the latter algbera is irreducible, Shur's lemma implies that the unique (up to multiplicative factors) solution to Eq. (77) is given by

$$
A=\mathbb{1} \quad \text { and } \quad \alpha_{\nu}=0 .
$$

We then find that the eigenvalue of $\mathbb{T}_{\pi / 2, \phi}[h]$ with maximal magnitude is 1 and corresponds to the unique left eigenvector

$$
\langle\mathbb{1}|=\frac{1}{2^{n t / 2}} \sum_{\left\{s_{\nu, \tau}\right\}}\left\langle\left\{s_{\nu, \tau}\right\}\right| \otimes\left\langle\left\{s_{\nu, \tau}\right\}\right|,
$$

where we use the computational basis, omit complex conjugation as the basis is real, and we include the normalization factor $\sqrt{\operatorname{tr}[\mathbb{1}]}=2^{n t / 2}$. Note that the unique right eigenvector of $\mathbb{1}_{\pi / 2, \phi}[h]$ associated with $\lambda=1$ is given by $|\mathbb{1}\rangle=(\langle\mathbb{1}|)^{\dagger}$, as it can be directly verified.

We also observe that since we just proved that Eqs. (65) and (66) have $\langle A|=\langle\mathbb{1}|$ as the only solution, and, moreover,

$$
\langle\mathbb{1}| \prod_{\nu=1}^{n} \mathbb{B}_{\nu, 1}^{z}[\theta] \neq \lambda_{\max }\langle\mathbb{1}|, \quad \theta \neq \pi / 2,
$$

the separated problem (64)-(66) has no solution for $\theta \neq \pi / 2$.

\section{B. Entanglement dynamics}

Our next step is to use the eigenvectors determined above to compute the entanglement dynamics. First, we note that the eigenvector $|\mathbb{1}\rangle$ is independent of $\phi$ and $h$. Moreover, $|1\rangle$ is orthogonal to all left generalized eigenvectors corresponding to the eigenvalues 0 of $\mathbb{T}_{\pi / 2, \phi}[h]$ for all $\phi$ and $h$. These two facts imply

$$
\lim _{L \rightarrow \infty} S_{A}^{(n)}(t)=\frac{1}{1-n} \log \left[\left\langle\Psi\left|\left(\prod_{j=1}^{N} \mathbb{T}_{\pi / 2, \phi_{j}}\left[h_{j}\right]\right)\right| \Psi\right\rangle\right],
$$

where we introduce

$$
|\Psi\rangle \equiv \mathbb{P}^{\dagger}|\mathbb{1}\rangle, \quad\langle\Psi| \equiv(|\Psi\rangle)^{\dagger}=\langle\mathbb{1}| \mathbb{P} .
$$

The relation (81) can be used to find the slope of the linear growth of the entanglement entropy. Indeed, taking $N$ to infinity we have

$$
\lim _{N \rightarrow \infty} \lim _{L \rightarrow \infty} S_{A}^{(n)}(t)=\frac{2}{1-n} \log |\langle\Psi \mid \mathbb{1}\rangle|=2 t \log 2 .
$$


The simple structure of $\mathbb{T}_{\pi / 2, \phi}[h]$, however, allows us to progress further and evaluate Eq. (81) exactly for each $N$.

This can be done by making use of the following remarkable identity

$$
\begin{aligned}
& \left\langle\Psi\left|\left(\prod_{j=1}^{N} \mathbb{T}_{\pi / 2, \phi_{j}}\left[h_{j}\right]\right)\right| \Psi\right\rangle \\
& \quad=\left\langle\Psi\left|\prod_{\nu=1}^{n}\left[\prod_{\tau=0}^{\lfloor N / 2\rfloor-1}\left[\mathbb{G}_{\nu, t-\tau}^{z} \mathbb{G}_{\nu, t-\tau}^{x}\right]\left[\mathbb{G}_{\nu, t-\lfloor N / 2]}^{z}\right]^{\bmod (N, 2)}\right]\right| \Psi\right\rangle, \\
& \forall \phi_{j}, h_{j},
\end{aligned}
$$

where $\lfloor\cdot\rfloor$ denotes the floor function. Here we adopt the convention

$$
\mathbb{G}_{\nu, \tau}^{a}=\mathbb{1}, \quad \tau \leq 0,
$$

and, to lighten the notation, from now on we assume that a product $\prod \cdots$ picks only a single factor on its right unless several terms are grouped within a square bracket $[\cdots]$.

The identity (84) is proven in Appendix F using the explicit form of $\mathbb{T}_{\pi / 2, \phi}[h]$, and the following useful properties of the state (82)

$$
\begin{aligned}
& \prod_{\nu=1}^{n} O_{\nu} \otimes O_{\nu}^{*}|\Psi\rangle=|\Psi\rangle, \\
& \langle\Psi| \prod_{\nu=1}^{n} O_{\nu} \otimes O_{\nu}^{*}=\langle\Psi|,
\end{aligned}
$$

where $O_{\nu}$ acts nontrivially as the unitary operator $O$ only on the $\nu$ th copy of $\mathcal{H}_{t}$ in $\mathcal{H}_{n t}$, i.e.,

$$
O_{\nu}=\mathbb{1}_{\mathcal{H}_{t}}^{\otimes(\nu-1)} \otimes O \otimes \mathbb{1}_{\mathcal{H}_{t}}^{\otimes(n-\nu)}
$$

These properties are proven in Appendix E.

A striking consequence of Eq. (84) is that the entanglement entropies evolving from separating states are completely independent of the configuration of longitudinal magnetic fields $\left\{h_{j}\right\}$ and of the initial-state angles $\left\{\phi_{j}\right\}$. For instance, this means that the same result is obtained in the integrable and in the nonintegrable case, with or without disorder.

The evaluation of the rhs of Eq. (84) is now straightforward. First, we note that in the computational basis (46) of $\mathcal{H}_{t}^{\otimes 2 n}$ we have

$$
\begin{aligned}
& \left\langle\left\{s_{\nu, \tau}^{\prime}\right\}\left|\otimes\left\langle\left\{r_{\nu, \tau}^{\prime}\right\}\right| \prod_{\nu=1}^{n}\left[\prod_{\tau=0}^{\lfloor N / 2\rfloor-1}\left[\mathbb{G}_{\nu, t-\tau}^{z} \mathbb{G}_{\nu, t-\tau}^{x}\right]\left[\mathbb{G}_{\nu, t-\lfloor N / 2]}^{z}\right]\right]^{\bmod (N, 2)}\right] \mid\left\{s_{\nu, \tau}\right\}\right\rangle \otimes\left|\left\{r_{\nu, \tau}\right\}\right\rangle
\end{aligned}
$$

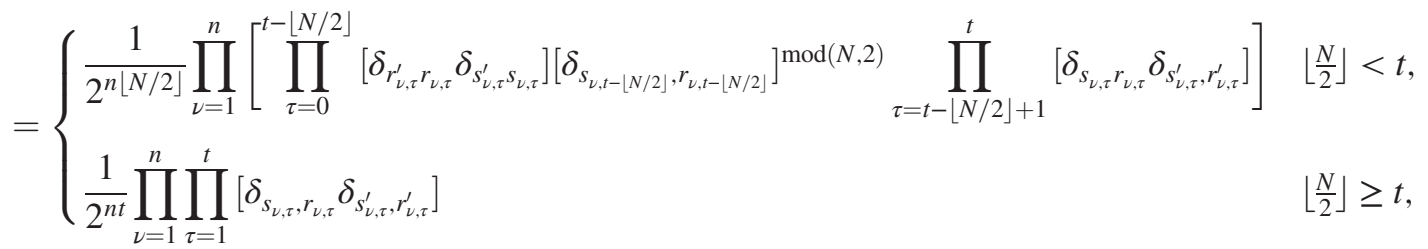

where the matrix elements of $\mathbb{G}_{\nu, \tau}^{x}$ and $\mathbb{G}_{\nu, \tau}^{z}$ are computed by repeated use of

$$
\begin{aligned}
& \left\langle s^{\prime}\left|\otimes\left\langle r^{\prime}|\mathbb{1}| s\right\rangle \otimes\right| r\right\rangle=\delta_{s, s^{\prime}} \delta_{r, r^{\prime}}, \\
& \left\langle s^{\prime}\left|\otimes\left\langle r^{\prime}\left|\frac{1}{2}\left(\mathbb{1}+\sigma^{z} \otimes \sigma^{z}\right)\right| s\right\rangle \otimes\right| r\right\rangle=\delta_{s, s^{\prime}} \delta_{r, r^{\prime}} \delta_{s, r}=\delta_{s, s^{\prime}} \delta_{s, r} \delta_{s^{\prime}, r^{\prime}}, \\
& \left\langle s^{\prime}\left|\otimes\left\langle r^{\prime}\left|\frac{1}{2}\left(\mathbb{1}+\sigma^{z} \otimes \sigma^{z}\right) \frac{1}{2}\left(\mathbb{1}+\sigma^{x} \otimes \sigma^{x}\right)\right| s\right\rangle \otimes\right| r\right\rangle=\frac{1}{2} \delta_{s, r} \delta_{s^{\prime}, r^{\prime}}, \quad s, r, s^{\prime}, r^{\prime} \in\{ \pm 1\} .
\end{aligned}
$$

Then, we plug Eq. (89) into the rhs of Eq. (84). For $t>\lfloor N / 2\rfloor$, we find

$$
\begin{aligned}
& \left.\langle\Psi| \prod_{\nu=1}^{n}\left[\prod_{\tau=0}^{\lfloor N / 2\rfloor-1}\left[\mathbb{G}_{\nu, t-\tau}^{z} \mathbb{G}_{\nu, t-\tau}^{x}\right]\left[\mathbb{G}_{\nu, t-\lfloor N / 2\rfloor}^{z}\right]\right]^{\bmod (N, 2)}\right]|\Psi\rangle \\
& =\frac{1}{2^{n\lfloor N / 2\rfloor+n t}} \sum_{\left\{s_{\nu, \tau}\right\}} \sum_{\left\{s_{\nu, \tau}^{\prime}\right\}} \prod_{\nu=1}^{n}\left[\prod_{\tau=1}^{t-\lfloor N / 2\rfloor}\left[\delta_{s_{\nu+1, \tau}^{\prime} s_{\nu+1, \tau}} \delta_{s_{\nu, \tau}^{\prime} s_{\nu, \tau}}\right]\left[\delta_{\left.s_{\nu, t-\lfloor N / 2\rfloor}, s_{\nu+1, t-\lfloor N / 2]}\right]} \bmod (N, 2) \prod_{\tau=t-\lfloor N / 2\rfloor+1}^{t}\left[\delta_{s_{\nu, \tau} s_{\nu+1, \tau}} \delta_{s_{\nu, \tau}^{\prime}, s_{\nu+1, \tau}^{\prime}}\right]\right]\right. \\
& =\frac{1}{2^{n\lfloor N / 2\rfloor+n t}}\left[\sum_{\left\{s_{\nu, t}\right\}_{\tau<t-\lfloor N / 2\rfloor}} 1\right]\left[\sum_{\left\{s_{\nu, t-\lfloor N / 2]}\right\}}\left[\delta_{s_{\nu, t-[N / 2]}, s_{\nu+1, t-\lfloor N / 2]}}\right] \bmod (N, 2)\right]\left[\sum_{\left\{s_{1, \tau}\right\}_{\tau>t-\lfloor N / 2\rfloor}} 1\right]^{2} \\
& =\frac{1}{2^{n\lfloor N / 2\rfloor+n t}} 2^{n(t-\lfloor N / 2\rfloor-1)} 2^{n-(n-1) \bmod (N, 2)} 2^{2\lfloor N / 2\rfloor}=2^{N(1-n)} .
\end{aligned}
$$


Proceeding analogously for $t \leq\lfloor N / 2\rfloor$, we have

$$
\left\langle\Psi\left|\prod_{\tau=0}^{\lfloor N / 2\rfloor-1}\left[\mathbb{G}_{\nu, t-\tau}^{z} \mathbb{G}_{\nu, t-\tau}^{x}\right]\left[\mathbb{G}_{\nu, t-\lfloor N / 2]}^{z}\right]^{\bmod (N, 2)}\right| \Psi\right\rangle=2^{2 t(1-n)} .
$$

Therefore, we finally obtain that for initial states in the class $\mathcal{T}$, all entanglement entropies $S_{A}^{(n)}(t)$ with $n=2,3, \ldots$ are exactly given by Eq. (23). This, however, implies that

$$
\operatorname{Spec}\left[\rho_{A}(t)\right]=\left\{2^{-\min (2 t, N)}, 0\right\},
$$

where $2^{-\min (2 t, N)}$ has multiplicity $2^{\min (2 t, N)}$, while 0 has multiplicity $2^{N-\min (2 t, N)}$. As a consequence, the result (23) holds for all $S_{A}^{(\alpha)}(t)$ with any real positive $\alpha$.

\section{ENTANGLEMENT SPREADING FROM GENERIC STATES}

The exact results derived in the previous sections have three remarkable features. (i) The entropies do not depend at all on the longitudinal magnetic fields. In particular, they are not affected by whether or not the system is integrable. (ii) The entropies grow at the maximal speed allowed by the range of the Hamiltonian and the dimension of the local Hilbert space [they saturate the minimal cut bound (24)]. (iii) At each fixed time $t$, all entanglement entropies coincide, signaling a flat entanglement spectrum, i.e., that all nonzero eigenvalues of the density matrix reduced to the block $A$ are equal.

It is interesting to wonder whether these are general features of the entanglement spreading in the self-dual kicked Ising chain or, instead, if they are special properties of separating initial states. In other words, it is interesting to ask whether the entanglement dynamics from separating states is an exceptional case or, even though special, it can be used to model the generic behavior. To this aim, in this section we consider the entanglement spreading from generic product states (12) which are not separating. In this case, as we point out above, we are unable to address the problem in a fully analytical fashion and we resort to a numerical analysis.

From the physical point of view, it is easy to see that the most convenient time regimes to examine possible modifications of the features (i)-(iii) are very different. Indeed, for $\boldsymbol{h} \neq \boldsymbol{0}$ the system is ergodic, and any finite subsystem is expected to relax to the infinite-temperature state irrespective of the initial conditions. This means all entropies are expected to saturate to the universal value $N \log 2$. On the other hand, for $\boldsymbol{h}=\mathbf{0}$ the system is integrable, and finite subsystems relax to generalized Gibbs ensembles [71,72]. We then expect the stationary values of the entropies to retain some memory of the initial configuration. To highlight the difference between integrable and nonintegrable systems, it is then convenient to focus on the "saturation regime" $t \sim N$, where the entropies become stationary. The generic relaxation to the infinite-temperature state, however, also means that to see some dependence of the entanglement spectrum on $\boldsymbol{h}$, or on the initial state, one has to stay away from the saturation regime and focus on the "growth regime" $t \ll N$. The latter is obviously also the regime of interest to study variations in the speed of entanglement growth.

The saturation regime can be easily accessed by a "direct" numerical approach. Namely, we consider a finite volume $L$ and determine the time-evolving state by means of the efficient time-propagation algorithm described in the Supplemental Material of Ref. [56]. The entanglement entropies are found by computing and diagonalizing the reduced density matrices $\rho_{A}(t)$ [cf. Eq. (14)] and using Eq. (15). Note that a similar numerical analysis, in the case of the von Neumann entropy, has been performed in Ref. [44].

Some representative examples of our results are reported in Fig. 7. First of all, we see that the qualitative behavior of the entanglement entropies is the same as that for separating states, both in the homogeneous (translationally invariant) and in the inhomogeneous case. The entropy grows in an approximately linear fashion until it saturates to a value proportional to the subsystem size. There is, however, a clear qualitative difference emerging between the integrable case and the generic one: In the generic case, the entropies always saturate to $N \log 2$ (minus the expected correction due to a finite $N / L[43,73,74])$, while this does not happen at the integrable point. In particular, in the inset of Fig. 8, we report the evolution of $S_{A}^{(2)}(t)$ for several homogeneous nonseparating initial states evolving under the integrable kicked Ising Hamiltonian. We see that, in contrast to the generic case, the saturation values depend on the initial state.

Interestingly, the evolution of the entropies shows very different finite-size effects in the integrable and nonintegrable cases. In the former case, the entropies start to decrease at times larger than $(L-N) / 2$, while in the latter case, they remain constant once they reach the saturation values. These behaviors, respectively, agree with the predictions of the quasiparticle and the minimal-membrane picture. Indeed, for $L>2 N$ the surface of the membrane is not affected by the system being finite. On the contrary, the quasiparticle picture predicts oscillations of the entropies due to quasiparticles traversing the entire system and going back to their initial positions. In particular, if the initial state is homogeneous, using that in our case the quasiparticles have all unit speed (and taking, for convenience, $L$ even), we find that the quasiparticle-picture prediction is $L / 2$ periodic and, for $t \in\{0,1, \ldots, L / 2\}$, it reads as

$$
S_{A}^{(\alpha)}(t)=\min (2 t, L-2 t, N) S_{\theta, \phi}^{(\alpha)},
$$




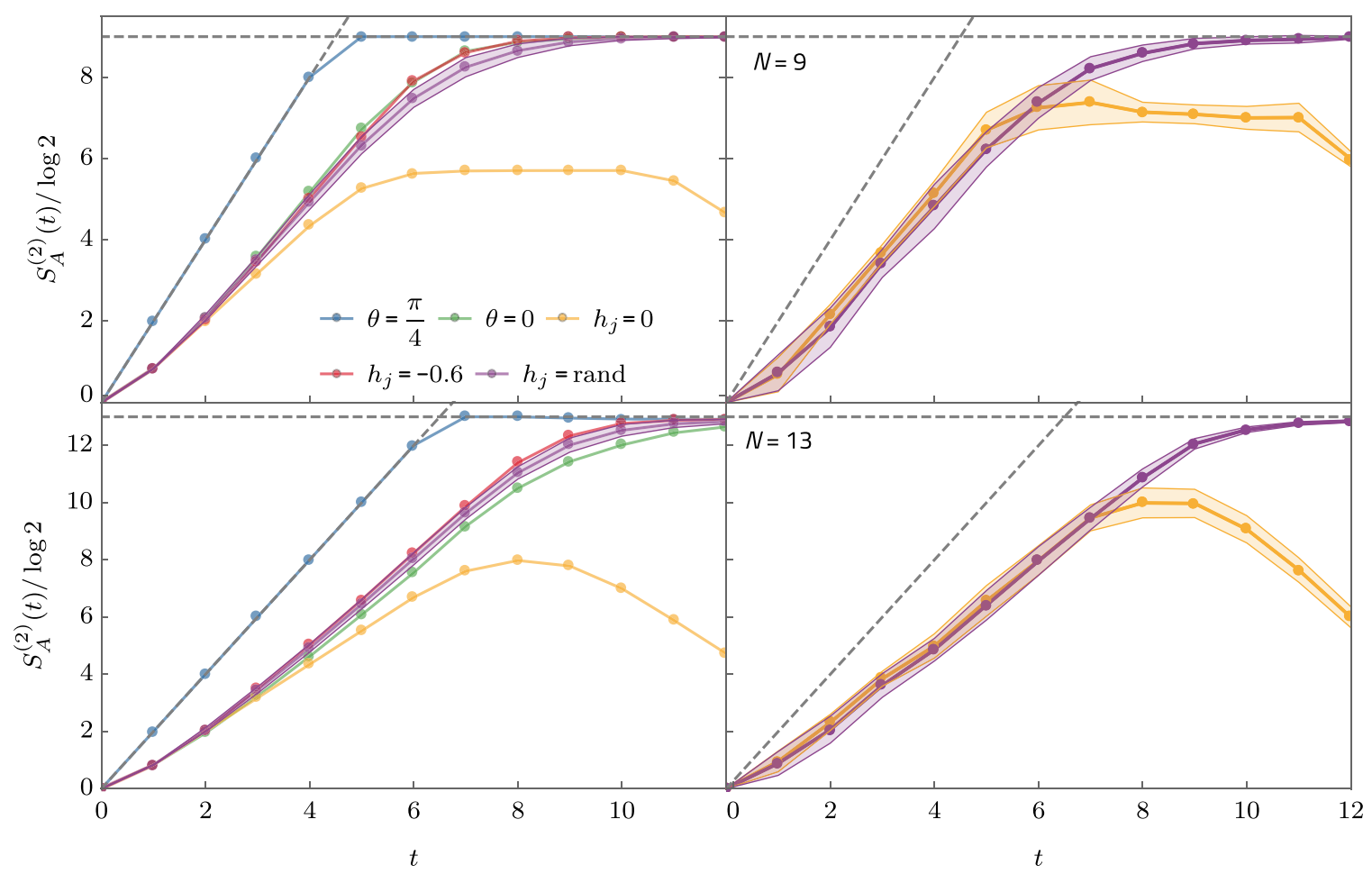

FIG. 7. The second Rényi entropy for a kicked Ising system of $L=30$ spins evolving from "tilted" initial states (12). Top and bottom two panels have, respectively, $N=9$ and $N=13$. The two panels on the left report results for translational-invariant initial states. The blue and green curves correspond, respectively, to transverse and longitudinal separating states [cf. Eqs. (17) and (18)]. Other curves correspond to the initial state $\theta_{j}=\phi_{j}=1$ and different magnetic fields as indicated in the legend. The two panels on the right correspond to the maximally disordered cases, where the spins at each site point in a random direction, and the magnetic fields $h_{j}$ are either random (purple) or zero (yellow). In the cases with random parameters, we show the average values for a sample of eight realizations using a continuous line and indicate a standard deviation of one realization by a shaded area.

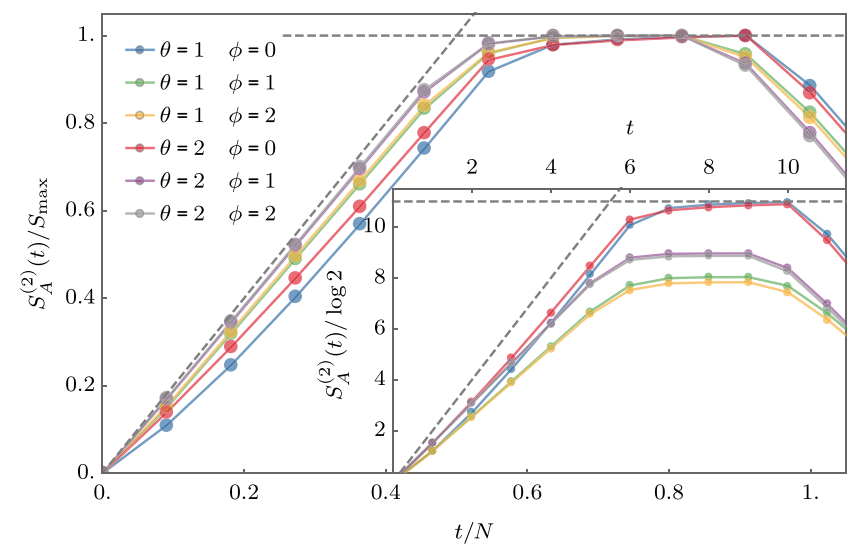

FIG. 8. Time evolution of the second Rényi entropy for a subsystem of $N=11$ spins in a kicked Ising system of $L=30$ at the integrable point $\boldsymbol{h}=\mathbf{0}$ for different translationally invariant initial states. The main panel shows the rescaled curves, which are close to Eq. (94) (black dashed line) given by the quasiparticle picture. In the inset, we show the nonrescaled version, where it is apparent that the saturation value depends on the initial state. Note a recurrence after the time $t=10$, consistent with the quasiparticle picture. where $S_{\theta, \phi}^{(\alpha)} \leq \log 2$ is a ( $N$ - and $L$-independent) constant. This prediction holds in the asymptotic limit $t, N \rightarrow \infty$ with fixed $t / N$, but, as shown in the main panel of Fig. 8, it is in fair agreement with our numerical results already for $N=11$. Note that, even if the system is free, $S_{\theta, \phi}^{(\alpha)}$ cannot be generically computed analytically. Indeed, for generic values of $\boldsymbol{\theta}$ and $\boldsymbol{\phi}$, the states are not Gaussian in terms of the time-evolving fermions, and this makes the problem analytically untreatable. Moreover, since the dispersion is linear, the usual arguments about Gaussification do not apply [75,76]. Interestingly, not even separating states are always Gaussian: Transverse separating states are Gaussian only for $\phi_{j}=0, \pi$ [77].

A natural question is what happens to the finite-size oscillations when the integrability is weakly broken? This is investigated in Fig. 9, which compares the behavior of the von Neumann entropy [cf. Eq. (16)] for increasing values of the (homogeneous) longitudinal magnetic field. We see that the finite-size oscillations become damped and disappear at large enough times. This can be interpreted as a sign of the decay of the quasiparticles. Consistently, the 


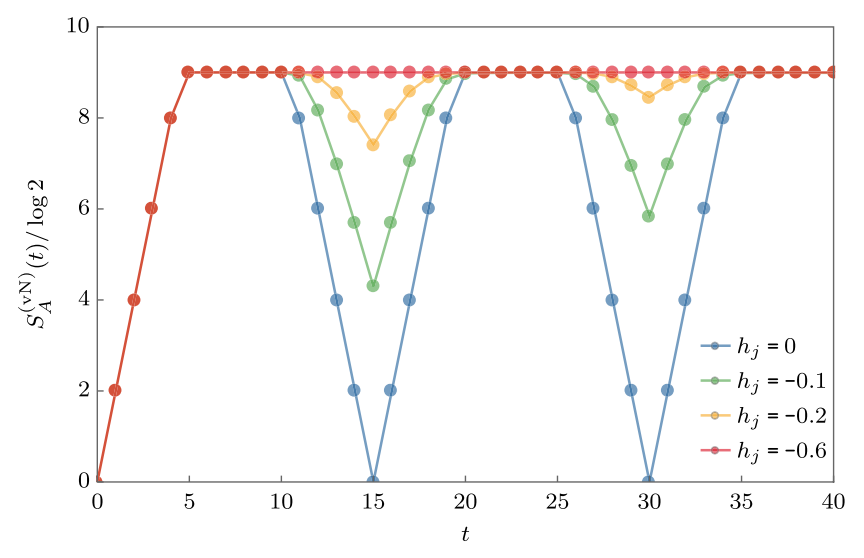

FIG. 9. The von Neumann entropy [cf. Eq. (16)] for a subsystem of $N=9$ spins in a kicked Ising system of $L=30$ evolving from the separating state with $\theta_{i}=\pi / 2$ and $\phi_{i}=0$ for different values of the longitudinal magnetic field.

decay speed increases with the magnitude of the longitudinal magnetic field. Moreover, for any fixed $\boldsymbol{h} \neq \mathbf{0}$, the peaks are observed to decay when the volume of the system increases.

Finally, we note that Fig. 7 also contains some information on the speed of entanglement growth. Indeed, we see that the time evolution of the entropies depends (although weakly for $\boldsymbol{h} \neq \mathbf{0}$ ) on the configuration of magnetic fields, indicating that the feature (ii) is lost at short times. It is, however, very hard to make any definitive statement based on Fig. 7. The direct numerical approach allows us to access the linear growth regime only for very short times, and it is impossible to exclude that (ii) remains as an asymptotic feature of the entanglement dynamics. A similar argument holds regarding the entanglement spectrum.

A useful way to circumvent this problem is offered by the expression for the Rényi entropies resulting from the duality mapping, namely, Eq. (60) (a similar duality-based approach has also been proposed in the context of tensor networks [78]). This expression becomes particularly convenient in the translational-invariant case

$$
h_{j}=h, \quad \theta_{j}=\theta, \quad \phi_{j}=\phi, \quad \forall j .
$$

Indeed, in this case one can use the general constraints (69) to take analytically the limit of infinite $L$ and $N$ and focus on the growth regime. Specifically, for $n=2,3, \ldots$, we find

$$
\lim _{N \rightarrow \infty} \lim _{L \rightarrow \infty} S_{A}^{(n)}(t)=\frac{2}{1-n} \log \left|\frac{\left\langle M_{L}|\mathbb{P}| M_{R}\right\rangle}{\left\langle M_{L} \mid M_{R}\right\rangle}\right|,
$$

where $\left\langle M_{L}\right|$ and $\left|M_{R}\right\rangle$ are, respectively, the left and right eigenstates of $\mathbb{T}_{\theta, \phi}[h]$ corresponding to the eigenvalue 1 . The constraints (69) imply that these vectors exist and are unique. Note that the simplification (96) cannot be generically performed in the inhomogeneous case, since transfer matrices with different $h, \theta$, and $\phi$ have different left and right eigenvectors.

The numerical evaluation of Eq. (96) is achieved in two steps. First, one has to determine the left and right eigenvectors and then evaluate the matrix element. Finding eigenvectors is particularly convenient due to the tensor product structure of the transfer matrix [cf. (51)]. Indeed, we can search eigenvectors of the form

$$
\left|M_{R}\right\rangle=\bigotimes_{\nu=1}^{n}\left|A_{R}\right\rangle, \quad\left\langle M_{L}\right|=\bigotimes_{\nu=1}^{n}\left\langle A_{L}\right|,
$$

where $\left|A_{R}\right\rangle,\left|A_{L}\right\rangle \in \mathcal{H}_{t} \otimes \mathcal{H}_{t}$. In the notation of Fig. 6, this means that we can effectively work in the Hilbert space of the $\nu$ th copy in both the positive-time and negative-time spaces (corresponding to the $\nu$ th column of Fig. 6). The eigenvectors are efficiently determined by means of a simple "power method": One starts from a random vector and finds $\left|A_{R}\right\rangle$ by repeated application of $\mathbb{T}_{\theta, \phi}^{(L)}[h]$. The left eigenvector $\left\langle A_{L}\right|$ is then determined by using $\left|A_{L}\right\rangle=e^{-i(\pi / 8) M_{1}^{x}} \otimes e^{i(\pi / 8) M_{1}^{x}}\left|A_{R}\right\rangle$. Proceeding in this way, the eigenvector can be determined in $\mathcal{O}\left(t m 2^{2 t}\right)$ operations, where $m$ is the number of iterations of the power method [79].

The form (97) is also convenient for evaluating the matrix element in Eq. (96). Indeed, after a straightforward calculation, we find

$$
\lim _{N \rightarrow \infty} \lim _{L \rightarrow \infty} S_{A}^{(n)}(t)=\frac{2}{1-n} \log \frac{\operatorname{tr}\left[\left(A_{R}^{\dagger} A_{L}\right)^{n}\right]}{\left[\operatorname{tr}\left(A_{R}^{\dagger} A_{L}\right)\right]^{n}},
$$

where $A_{L, R}$ are the $2^{t} \times 2^{t}$ matrices corresponding to the vectors $\left\langle A_{R, L}\right|$ through the vector-to-operator mapping (73) (performed for $n=1$ ). Note that for transverse separating initial states, we have

$$
A_{R}=A_{L} \propto \mathbb{1},
$$

but for generic initial states, these matrices become nontrivial. The rhs of Eq. (98) can be numerically evaluated for integer $n \geq 2$, whereas Rényi entropies with more general index $\alpha>0$ can be found by analytically continuing Eq. (98) in $n$ and diagonalizing $A_{R}^{\dagger} A_{L}$ numerically to compute the powers. Evaluating Eq. (98) has complexity $\propto 2^{3 t}$ and is the bottleneck of the numerical procedure, meaning that we are able to reach up to $t_{\max }=17$.

We stress that, since the constraints (69) hold also away from the self-dual points, this procedure can be used to study the entanglement spreading in the entire parameter space of the kicked Ising model. Note that close enough to the self-dual points (and to separating initial states) an analytical perturbative analysis is also possible. These aspects, however, go beyond the scope of the present manuscript and will be investigated in the course of future 


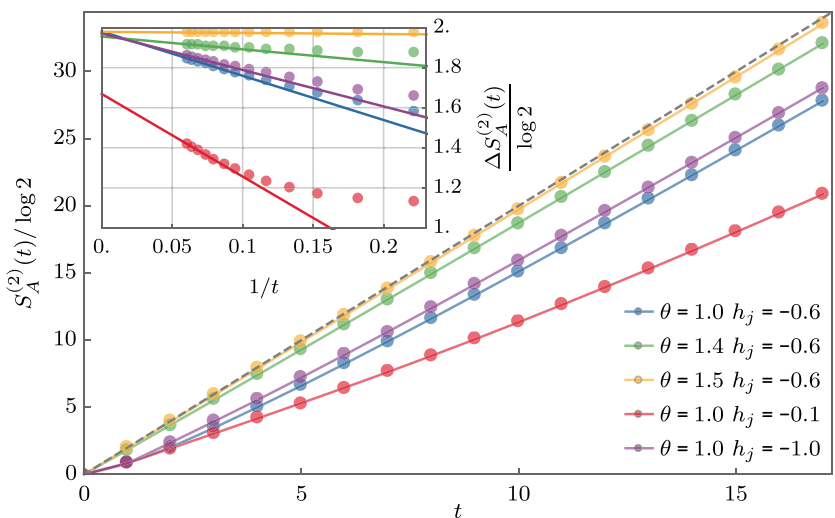

FIG. 10. The second Rényi entropy for a kicked Ising system evolving from "tilted" initial states (12) in the thermodynamic limit. The colored lines correspond to nonseparating initial states (we take $\phi=\theta$, with $\theta$ and $h$ specified in the legend) and are determined numerically evaluating Eq. (98), while the gray dashed lines report, for comparison, the result from separating states. The inset shows the instantaneous slope $\Delta S_{A}^{(2)}(t)$ [cf. Eq. (100)] as a function of $1 / t$. The points are computed by evaluating Eq. (98), while the lines are a linear fit of the last two points.

research. Here we focus on the self-dual points (5) and use this duality-based numerical approach to effectively investigate the fate of the features (ii) and (iii) when the system is initialized in a generic state (12).

Representative examples of our numerical results are reported in Figs. 10 and 11. We see that, consistent with the results in Fig. 7, at short times both (ii) and (iii) are violated. The entropies grow in an approximately linear fashion, but the slope appears to depend on the initial state and on the longitudinal magnetic field $h$ (see Fig. 10). Moreover, different Rényi entropies have different slopes (see Fig. 11). Crucially, however, a more refined analysis suggests that these deviations vanish for large times. To show this, we proceed as follows. First, we introduce the "instantaneous" slopes

$$
\Delta S_{A}^{(\alpha)}(t-1 / 2) \equiv S_{A}^{(\alpha)}(t)-S_{A}^{(\alpha)}(t-1) .
$$

Computing these quantities numerically, we observe that they become linear functions of $1 / t$ for large enough times. We then perform a linear fit in $1 / t$ and extrapolate the result to $t=\infty$. In the integrable case, this procedure gives results consistent with the quasiparticle picture prediction [cf. Eq. (94)], namely,

$$
\left.\Delta S_{A}^{(\alpha)}(\infty)\right|_{h=0}=2 S_{\theta, \phi}^{(\alpha)} \leq 2 \log 2 .
$$

Instead, in the nonintegrable case the results are consistent with

$$
\left.\Delta S_{A}^{(\alpha)}(\infty)\right|_{h \neq 0}=2 \log 2
$$

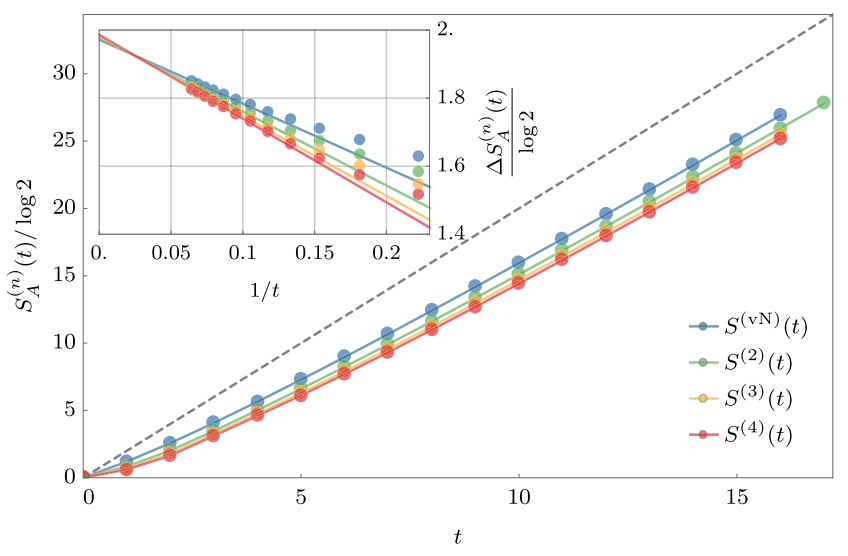

FIG. 11. Time evolution of different Rényi entropies in a kicked Ising system with longitudinal magnetic field $h=-0.6$ in the thermodynamic limit. The initial state is of the form (12) with $\theta=\phi=1$. The colored lines correspond to Renyi indices $n=1$, 2, 3, 4 and are determined numerically evaluating Eq. (98), while the gray dashed line reports, for comparison, the result from separating states. The inset shows the instantaneous slope $\Delta S_{A}^{(n)}(t)$ [cf. Eq. (100)] as a function of $1 / t$. The points are computed by evaluating Eq. (98), while the lines are linear extrapolations from the last two data points.

This behavior is observed for any initial state (12), for any nonvanishing longitudinal magnetic field, and for any Rényi index $\alpha$, as we demonstrate in the insets of Figs. 10 and 11. The only seemingly exceptional cases are observed when the system is very close to the integrable point (see, e.g., the red curve in the inset of Fig. 10). This is, however, straightforwardly explained as a prethermalization effect [80-82]. For small enough longitudinal fields, there is an initial transient before the quasiparticles decay in which the observables follow the integrable predictions. After this transient, however, the entropies are expected to follow the nonintegrable curves, reaching the asymptotic value (102) for the slope. For instance, this is consistent with the behavior of the red curve in the inset of Fig. 10. Interestingly, since $S_{\theta, \phi}^{(\alpha)}=\log 2$ for separating states, this effect is not observed in our exact result (23). From the physical point of view, (102) is very natural. Since the system is ergodic, the initial-state dependence is washed away at large enough times, and the entropies behave as if they would be evolving from separating states. We stress that, since we are dealing with a Floquet system with no local conservation laws, the entanglement spreading is expected to be totally independent of the initial state: It should not depend even on its energy which is not conserved during the time evolution. As expected, this does not happen in the integrable case.

In conclusion, the numerical results presented in this section support the following general picture. The evolution of the entanglement entropies from a generic initial state (12) in the thermodynamic limit differs from that from 
separating states [cf. Eq. (23)] and depends explicitly on the initial state, the longitudinal magnetic fields, and the Rényi numbers. In the scaling limit $t, N \rightarrow \infty$; however, all these dependences are washed away: The entropies collapse to the prediction (23) if the system is nonintegrable and to the thermodynamic limit of Eq. (94) if the system is integrable. In other words, our exact result (23) serves as an asymptotic description of the entanglement spreading in the nonintegrable kicked Ising model. This picture is also supported by the numerical results of Ref. [44], which found that the evolution of the von Neumann entropy averaged over all separable initial states is consistent with Eq. (23). Finally, we remark that this section also demonstrates that extracting universal information on the behavior of the entanglement entropies from the numerics is extremely hard, especially in the ergodic case where the entanglement growth is exceptionally fast. This highlights even more the practical importance of our exact result (23).

\section{CONCLUSIONS}

We develop a constructive and mathematically rigorous approach for computing the dynamics of bipartite entanglement in a class of "maximally scrambling," locally interacting, chaotic spin chains. Specifically, we consider the so-called self-dual kicked Ising spin chains, where the integrability is broken by switching on an external longitudinal magnetic field. We prepare the system in a class of ground states of simple local Hamiltonians and determine exactly the dynamics of all Rényi entropies of finite blocks of spins of arbitrary size. The results presented are nonperturbative, no kind of averaging is involved, and, most importantly, they hold in the presence of longitudinal magnetic fields with arbitrary spatial dependence. It is remarkable that such an explicit exact calculation can be performed for a specific nonintegrable many-body system.

Our result shows that in the thermodynamic limit, the Rényi entropies of finite blocks of spins are independent of the longitudinal magnetic field at all times. Moreover, they obey universal scaling laws that can be predicted both by means of the quasiparticle picture of Ref. [15], put forward for integrable models, and of the minimal membrane picture of Ref. [50] propounded for generic systems.

Using our novel rigorous approach, we also develop a numerical procedure for studying the entanglement spreading from generic product initial states. A thorough numerical analysis suggests that, away from the integrable point, our exact result continues to describe the entanglement spreading at the leading order in time. On the contrary, in the integrable case, the entanglement production is generically renormalized by an initial-state-dependent multiplicative coefficient. Further qualitative differences between the integrable and the nonintegrable case emerge for finite systems. In particular, we show numerically that there are recurrences in the integrable case, which are absent in the nonintegrable one. We stress that these differences are correctly accounted for by the quasiparticle and minimal membrane pictures, which disagree for finite sizes.

Our analytical method can be used to highlight qualitative differences in the entanglement spreading of integrable and nonintegrable systems directly in the thermodynamic limit. To do that, one could follow Refs. [35,36] and consider the bipartite entanglement of disjoint blocks. Our preliminary results suggest that the scaling forms produced in the two cases are indeed different and, respectively, agree with the predictions of quasiparticle and membrane pictures. Another possible direction is to perturb the kicked Ising spin chains away from the self-dual points, where the predictions of the two pictures disagree also for the entanglement of a single block. This could be tested within our approach by using perturbation theory.

More generally, we expect that our method will allow for explicit calculations similar to the ones presented also for other measures of correlations and dynamical complexity, such as operator space entanglement entropy and out-oftime order correlators.

Finally, we believe that the remarkable algebraic structure unveiled in this work paves the way for the determination of a new class of exactly solvable, maximally chaotic models. Elements of this class can serve as minimal models for characterizing the nonequilibrium dynamics in generic systems.

\section{ACKNOWLEDGMENTS}

We thank Adam Nahum for very valuable comments on the manuscript and Pasquale Calabrese for stimulating discussions. We also wish to thank the two anonymous referees for their helpful and constructive comments that greatly contributed to improve the quality of the paper (especially that of Sec. VII). B. B. and T. P. acknowledge the hospitality of the Erwin Schrödinger Institute, Vienna, where this project has been conceived. The work is supported by Advanced Grant of European Research Council, Grant No. 694544-OMNES and the program P1-0402 of the Slovenian Research Agency.

\section{APPENDIX A: DUALITY OF TRACES}

Here we explicitly demonstrate the duality relation (27). Writing $\operatorname{tr}\left[\left(U_{K I}[\boldsymbol{h}]\right)^{t}\right]$ in the computational basis $\mathcal{B}_{L}$ [cf. Eq. (7)], we have 


$$
\begin{aligned}
\operatorname{tr}\left[\left(U_{K I}[\boldsymbol{h}]\right)^{t}\right]= & \sum_{\left\{\boldsymbol{s}_{\tau}\right\}}\left\langle\boldsymbol{s}_{1}\left|U_{K I}[\boldsymbol{h}]\right| \boldsymbol{s}_{t}\right\rangle\left\langle\boldsymbol{s}_{t}\left|U_{K I}[\boldsymbol{h}]\right| \boldsymbol{s}_{t-1}\right\rangle \cdots\left\langle\boldsymbol{s}_{2}\left|U_{K I}[\boldsymbol{h}]\right| \boldsymbol{s}_{1}\right\rangle \\
= & \left(\frac{\sin 2 b}{2 i}\right)^{(L t) / 2} \sum_{\left\{s_{\tau, j}\right\}}\left\{\exp \left[-i \tilde{J} \sum_{j=1}^{L} s_{1, j} s_{t, j}-i J \sum_{j=1}^{L} s_{t, j} s_{t, j+1}-i \sum_{j=1}^{L} h_{j} s_{t, j}\right]\right. \\
& \times \exp \left[-i \tilde{J} \sum_{j=1}^{L} s_{t, j} s_{t-1, j}-i J \sum_{j=1}^{L} s_{t-1, j} s_{t-1, j+1}-i \sum_{j=1}^{L} h_{j} s_{t-1, j}\right] \\
\vdots & \left.\times \exp \left[-i \tilde{J} \sum_{j=1}^{L} s_{2, j} s_{1, j}-i J \sum_{j=1}^{L} s_{1, j} s_{1, j+1}-i \sum_{j=1}^{L} h_{j} s_{1, j}\right]\right\}
\end{aligned}
$$

Here, $s_{\tau, L+j} \equiv s_{\tau, j}$, and in the second step we use the identity

$$
\left\langle s\left|e^{-i b \sigma^{x}}\right| r\right\rangle=\sqrt{\frac{\sin 2 b}{2 i}} \exp [-i \tilde{J} s r], \quad s, r \in\{ \pm 1\},
$$

where

$$
\tilde{J}=-\frac{\pi}{4}-\frac{i}{2} \log \tan b
$$

This expression can be thought of as the partition function of a two-dimensional Ising model with complex couplings on an $L \times t$ periodic lattice. In other words, the rhs of Eq. (A1) is proportional to the partition function of a classical statistical mechanical model with configuration energy given by

$$
\mathcal{E}\left[\left\{s_{\tau, j}\right\}, \boldsymbol{h}\right]=-\sum_{\tau=1}^{t} \sum_{j=1}^{L}\left(i J s_{\tau, j} s_{\tau, j+1}+i \tilde{J} s_{\tau, j} s_{\tau+1, j}+i h_{j} s_{\tau, j}\right) .
$$

Reorganizing the sum on the rhs of Eq. (A1), we also have

$$
\begin{aligned}
\operatorname{tr}\left[\left(U_{K I}[\boldsymbol{h}]\right)^{t}\right]= & \left(\frac{\sin 2 b}{2 i}\right)^{[(L t) / 2]} \sum_{\left\{s_{\tau, j}\right\}}\left\{\exp \left[-i \tilde{J} \sum_{\tau=1}^{t} s_{\tau, 1} s_{\tau+1,1}-i J \sum_{\tau=1}^{t} s_{\tau, 1} s_{\tau, L}-i \sum_{\tau=1}^{t} h_{1} s_{\tau, 1}\right]\right. \\
& \times \exp \left[-i \tilde{J} \sum_{\tau=1}^{t} s_{\tau, 2} s_{\tau+1,2}-i J \sum_{\tau=1}^{t} s_{\tau, 1} s_{\tau, 2}-i \sum_{\tau=1}^{t} h_{2} s_{\tau, 2}\right] \\
& \vdots \\
& \left.\times \exp \left[-i \tilde{J} \sum_{\tau=1}^{t} s_{\tau, L} s_{\tau+1, L}-i J \sum_{\tau=1}^{t} s_{\tau, L} s_{\tau, L-1}-i \sum_{\tau=1}^{t} h_{L} s_{\tau, L}\right]\right\},
\end{aligned}
$$

where we define $s_{t+\tau, j} \equiv s_{\tau, j}$. Using again the identity (A2), we finally find

$$
\operatorname{tr}\left[\left(U_{K I}[\boldsymbol{h}]\right)^{t}\right]=\operatorname{tr}\left[\tilde{U}_{K I}\left[h_{1} \tilde{\mathbf{1}}\right] \cdots \tilde{U}_{K I}\left[h_{L} \tilde{\mathbf{1}}\right]\right]
$$

where the "tilded" bold symbols denote vectors of $t$ components, and we introduce the dual transfer matrix

$$
\tilde{U}_{K I}[\tilde{\boldsymbol{h}}]=e^{-i \tilde{H}_{K}} e^{-i \tilde{H}_{I}[\tilde{\boldsymbol{h}}]},
$$


with

$$
\tilde{H}_{I}[\tilde{\boldsymbol{h}}] \equiv \tilde{J} \sum_{j=1}^{t} \sigma_{j}^{z} \sigma_{j+1}^{z}+\sum_{j=1}^{t} h_{j} \sigma_{j}^{z}, \quad \tilde{H}_{K} \equiv \tilde{b} \sum_{j=1}^{t} \sigma_{j}^{x}
$$

\section{APPENDIX B: SIMPLIFIED TRANSFER MATRIX FOR LONGITUDINAL SEPARATING STATES}

When the initial state is in the class $\mathcal{L}$ [cf. Eq. (18)], namely, when

$$
\theta_{j}=\bar{\theta}_{j} \equiv\left(1+s_{j}\right) \pi / 2, \quad s_{j} \in\{-1,1\}, \quad j \in\{1,2, \ldots, L\},
$$

the form (60) can be simplified by effectively reducing the dimension of the space where the trace acts. To see this, we note that in this case $\mathbb{B}_{\nu, 1}^{z}[\theta]$ becomes proportional to a projector

$$
\mathbb{B}_{\nu, 1}^{z}\left[\left(1+s_{j}\right) \pi / 2\right]=2 P_{\nu, 1}^{z, s_{j}} \otimes P_{\nu, 1}^{z, s_{j}},
$$

so that we have

$$
\begin{aligned}
\operatorname{tr} & {\left[\left(\prod_{j=1}^{N} \mathbb{T}_{\bar{\theta}_{j}, \phi_{j}}\left[h_{j}\right]\right) \mathbb{P}\left(\prod_{j=N+1}^{L} \mathbb{T}_{\bar{\theta}_{j}, \phi_{j}}\left[h_{j}\right]\right) \mathbb{P}^{\dagger}\right] } \\
& =2^{L n} \operatorname{tr}\left[\prod_{j=1}^{L} \prod_{\nu=1}^{n} P_{\nu, 1}^{z, s_{j}} e^{i(\pi / 4) \sigma_{\nu, 1}^{r}} \otimes P_{\nu, 1}^{z, s_{j}} e^{-i(\pi / 4) \sigma_{\nu, 1}^{r}}\right] \operatorname{tr}\left[\left(\prod_{j=1}^{N} \overline{\mathbb{T}}_{\pi / 2, \bar{\theta}_{j}-\pi / 2}\left[h_{j}\right]\right) \overline{\mathbb{P}}\left(\prod_{j=N+1}^{L} \overline{\mathbb{T}}_{\pi / 2, \bar{\theta}_{j}-\pi / 2}\left[h_{j}\right]\right) \overline{\mathbb{P}}^{\dagger}\right],
\end{aligned}
$$

where we introduce

$$
\begin{gathered}
\overline{\mathbb{P}} \equiv \mathbb{1} \otimes \prod_{\nu=1}^{n} \prod_{\tau=2}^{t} P_{(\nu, \tau),(\nu-1, \tau)}, \\
\overline{\mathbb{T}}_{\theta, \phi}[h] \equiv \mathbb{B}_{\nu, 2}^{z}[\theta] \cdot \mathbb{G}_{\nu, t}^{z} \cdot \overline{\mathbb{U}}_{\phi}[h] .
\end{gathered}
$$

Here the matrix $\bar{\cup}_{\phi}^{(\nu)}[h]$ is defined as

$$
\bar{\cup}_{\phi}^{(\nu)}[h] \equiv\left(\bar{U}_{\nu, \phi} \otimes \bar{U}_{\nu, \phi}^{*}\right) \cdot\left(e^{-i h \bar{M}_{\nu}^{z}} \otimes e^{i h \bar{M}_{\nu}^{z}}\right) \cdot\left(e^{i(\pi / 4) \bar{M}_{\nu}^{x}} \otimes e^{-i(\pi / 4) \bar{M}_{\nu}^{x}}\right),
$$

and the barred operators read as

$$
\bar{U}_{\nu, \phi} \equiv \exp \left[-\frac{i \pi}{4} \sum_{\tau=2}^{t-1} \sigma_{\nu, \tau}^{z} \sigma_{\nu, \tau+1}^{z}-i \frac{\phi}{2} \sigma_{\nu, 2}^{z}\right], \quad \bar{M}_{\nu}^{a} \equiv \sum_{\tau=2}^{t} \sigma_{\nu, \tau}^{a} .
$$

So, they have the same form as Eqs. (56) and (57), but at fixed $\nu$ they act nontrivially only in the space $\mathcal{H}_{t-1}$ composed of the last $t-1$ sites of $\mathcal{H}_{t}$. In other words, $\overline{\mathbb{T}}_{\theta, s}[h]$ has the same form as $\mathbb{T}_{\theta, s}[h]$ but acts on $\mathcal{H}_{t-1}^{\otimes 2 n}$ instead of $\mathcal{H}_{t}^{\otimes 2 n}$. We stress that the trace operations in expression (B3) and below are taken in the subspaces where the operators act nontrivially, for example, for the barred operators in $\mathcal{H}_{t-1}^{\otimes 2 n} \cong \mathcal{H}_{2 n(t-1)}$. Noting

$$
2^{L n} \operatorname{tr}\left[\prod_{j=1}^{L} \prod_{\nu=1}^{n} P_{\nu, 1}^{z, s_{j}} e^{i(\pi / 4) \sigma_{\nu, 1}^{x}} \otimes P_{\nu, 1}^{z, s_{j}} e^{-i(\pi / 4) \sigma_{\nu, 1}^{x}}\right]=2^{L n} \mid \operatorname{tr}\left[\left.\prod_{j=1}^{L}\left[P_{1,1}^{z, s_{j}} e^{\left.i(\pi / 4) \sigma_{1,1}^{x}\right]}\right]\right|^{2 n}=1, \quad \forall s_{j} \in\{-1,+1\},\right.
$$

we finally find 


$$
S_{A}^{(n)}(t)=\frac{1}{1-n} \log \operatorname{tr}\left[\left(\prod_{j=1}^{N} \overline{\mathbb{T}}_{(\pi / 2), \bar{\theta}_{j}-(\pi / 2)}\left[h_{j}\right]\right) \overline{\mathbb{P}}\left(\prod_{j=N+1}^{L} \overline{\mathbb{T}}_{(\pi / 2), \bar{\theta}_{j}-(\pi / 2)}\left[h_{j}\right]\right) \overline{\mathbb{P}}^{\dagger}\right]
$$

Therefore, we see that in this case the entropies are given by an expression of the form (60), with $\theta_{j}=\pi / 2$ and $\phi_{j}=(\pi / 2) s_{j}$ but with matrices acting on $\mathcal{H}_{t-1}^{\otimes 2 n}$ instead of $\mathcal{H}_{t}^{\otimes 2 n}$. Note that for $\theta_{j}=\bar{\theta}_{j}$, the states (12) do not depend on $\phi_{i}$, and this independence is correctly reflected in Eq. (B9).

\section{APPENDIX C: PROOF OF PROPERTY 1}

In this Appendix, we provide the proof of Property 1.

Proof.-For each state $\langle A|$, we have

$$
\left\langle A\left|\mathbb{T}_{\theta, \phi}[h] \mathbb{T}_{\theta, \phi}^{\dagger}[h]\right| A\right\rangle=\left\langle A\left|\prod_{\nu=1}^{n} \mathbb{B}_{\nu, 1}^{z}[\theta] \prod_{\nu=1}^{n} \mathbb{G}_{\nu, t}^{z} \prod_{\nu=1}^{n} \mathbb{B}_{\nu, 1}^{z}[\theta]\right| A\right\rangle \leq\left\langle A\left|\prod_{\nu=1}^{n} \mathbb{B}_{\nu, 1}^{z}[\theta]^{2}\right| A\right\rangle,
$$

where we use that $\mathbb{G}_{\nu, t}^{z}$ is a projector, so its expectation value on a normalized state is smaller or equal to 1 . Expanding $\mathbb{B}_{\nu, 1}^{z}[\theta]^{2}$, we then have

$$
\begin{aligned}
\left\langle A\left|\mathbb{T}_{\theta, \phi}[h] \mathbb{T}_{\theta, \phi}^{\dagger}[h]\right| A\right\rangle \leq & 4^{n}\langle A| \prod_{\nu=1}^{n}\left[\cos ^{4}(\theta / 2) P_{\nu, 1}^{z,+} \otimes P_{\nu, 1}^{z,+}+\sin ^{2}(\theta / 2) \cos ^{2}(\theta / 2) P_{\nu, 1}^{z,-} \otimes P_{\nu, 1}^{z,+}\right. \\
& \left.+\sin ^{2}(\theta / 2) \cos ^{2}(\theta / 2) P_{\nu, 1}^{z,+} \otimes P_{\nu, 1}^{z,-}+\sin ^{4}(\theta / 2) P_{\nu, 1}^{z,-} \otimes P_{\nu, 1}^{z,-}\right]|A\rangle .
\end{aligned}
$$

Since $P_{\nu, 1}^{z, \pm} \otimes P_{\nu, 1}^{z, \pm}$ are orthogonal projectors, we have

$$
\left\langle A\left|\mathbb{T}_{\theta, \phi}[h] \mathbb{T}_{\theta, \phi}^{\dagger}[h]\right| A\right\rangle \leq 4^{n} \max \left[\sin ^{4 n}(\theta / 2), \cos ^{4 n}(\theta / 2)\right] .
$$

In particular, choosing $\langle A|$ to be the left eigenstate of $\mathbb{T}_{\theta, \phi}[h]$ corresponding to the eigenvalue $\lambda$, we have

$$
|\lambda| \leq 2^{n} \max \left[\sin ^{2 n}(\theta / 2), \cos ^{2 n}(\theta / 2)\right]=(1+|\cos \theta|)^{n}=\lambda_{\max },
$$

which proves the first part of the claim.

To prove the point (ii a), we proceed by reductio ad absurdum. Suppose that the Jordan block of $\lambda$ is nontrivial: Let $\langle A|$ be the eigenvector associated with $\lambda$, and let $\langle B|$ be the first generalized eigenvector. As it is always possible, we choose $\langle B|$ to be normalized and orthogonal to $\langle A|$ (which is also normalized). We then have

$$
\langle B| \mathbb{T}_{\theta, \phi}[h]=\lambda\langle B|+x\langle A|, \quad x \neq 0 .
$$

This relation implies

$$
\left\langle B\left|\mathbb{T}_{\theta, \phi}[h] \mathbb{\mathbb { T }}_{\theta, \phi}^{\dagger}[h]\right| B\right\rangle=\left|\lambda_{\max }\right|^{2}+|x|^{2},
$$

which is impossible because it contradicts Eq. (C3). Point (ii b) follows by noting that in order to have the equality sign in Eq. (C3), we must have

$$
\langle A| \prod_{\nu=1}^{n} \mathbb{B}_{\nu, 1}^{z}[\theta]=\lambda_{\max }\langle A|
$$

$$
\langle A| \prod_{\nu=1}^{n} \mathbb{G}_{\nu, t}^{z}=\langle A| .
$$

Using now that $\langle A|$ is a left eigenvector of $\mathbb{T}_{\theta, \phi}[h]$, we have Eq. (66). This concludes the proof.

\section{APPENDIX D: SIMPLIFIED COMMUTATION RELATIONS}

In this Appendix, we prove the following property.

Property 2: The commutation relations (75) and (76) imply

$$
\begin{aligned}
A \sigma_{\nu, \tau}^{a} & =\sigma_{\nu, \tau}^{a} A, \quad \forall a \in\{x, y, z\}, \\
\tau & \in\{1, \ldots, t\}, \quad \nu \in\{1, \ldots, n\} .
\end{aligned}
$$


Proof.-First of all, we note that multiplying Eq. (76) on the left and on the right by $e^{-i(\pi / 4) M_{\nu}^{x}} e^{i h M_{\nu}^{z}} U_{\nu, \phi}^{\dagger}$ we have

$$
\begin{aligned}
A e^{-i(\pi / 4) M_{\nu}^{x}} e^{i h M_{\nu}^{z}} U_{\nu, \phi}^{\dagger} & =e^{i \alpha_{\nu}} e^{-i(\pi / 4) M_{\nu}^{x}} e^{i h M_{\nu}^{z}} U_{\nu, \phi}^{\dagger} A, \\
\alpha_{\nu} & \in \mathbb{R}, \quad \forall \nu \in\{1, \ldots, n\} .
\end{aligned}
$$

Using the conditions (75), (76), and (D2), we see that $A$ commutes with

$$
\begin{gathered}
e^{-i(\pi / 4) M_{\nu}^{x}} e^{i h M_{\nu}^{z}} U_{\nu, \phi}^{\dagger} \sigma_{\nu, t}^{z} U_{\nu, \phi} e^{-i h M_{\nu}^{z}} e^{i(\pi / 4) M_{\nu}^{x}} \\
=e^{-i(\pi / 4) M_{\nu}^{x}} \sigma_{\nu, t}^{z} e^{i(\pi / 4) M_{\nu}^{x}}=-\sigma_{\nu, t}^{y} .
\end{gathered}
$$

Indeed, we have

$$
\begin{aligned}
& A e^{-i(\pi / 4) M_{\nu}^{x}} e^{i h M_{\nu}^{z}} U_{\nu, \phi}^{\dagger} \sigma_{\nu, t}^{z} U_{\nu, \phi} e^{-i h M_{\nu}^{z}} e^{i(\pi / 4) M_{\nu}^{x}} \\
& \quad=e^{i \alpha_{\nu}} e^{-i(\pi / 4) M_{\nu}^{x}} e^{i h M_{\nu}^{z}} U_{\nu, \phi}^{\dagger} A \sigma_{\nu, t}^{z} U_{\nu, \phi} e^{-i h M_{\nu}^{z}} e^{i(\pi / 4) M_{\nu}^{x}} \\
& =e^{i \alpha_{\nu}} e^{-i(\pi / 4) M_{\nu}^{x}} e^{i h M_{\nu}^{z}} U_{\nu, \phi}^{\dagger} \sigma_{\nu, t}^{z} A U_{\nu, \phi} e^{-i h M_{\nu}^{z}} e^{i(\pi / 4) M_{\nu}^{x}} \\
& =e^{-i(\pi / 4) M_{\nu}^{x}} e^{i h M_{\nu}^{z}} U_{\nu, \phi}^{\dagger} \sigma_{\nu, t}^{z} U_{\nu, \phi} e^{-i h M_{\nu}^{z}} e^{i(\pi / 4) M_{\nu}^{x}} A,
\end{aligned}
$$

where in the first step we use Eq. (D2), in the second Eq. (76), and in the third Eq. (75). Using Eq. (75), we then have that $A$ also commutes with

$$
-i \sigma_{\nu, t}^{y} \sigma_{\nu, t}^{z}=\sigma_{\nu, t}^{x}
$$

We then have

$$
\left[A, \sigma_{\nu, t}^{a}\right]=0, \quad \forall a \in\{x, y, z\}, \quad \nu \in\{1, \ldots, n\}
$$

Using Eqs. (D6), (76), and (D2), we can then conclude the proof by induction.

We prove that if

$$
\begin{aligned}
{\left[A, \sigma_{\nu, \tau}^{a}\right] } & =0, \quad \forall a \in\{x, y, z\}, \\
\tau & \in\{\bar{\tau}+1, \bar{\tau}+2, \ldots, t\},
\end{aligned}
$$

then

$$
\left[A, \sigma_{\nu, \bar{\tau}}^{a}\right]=0, \quad \forall a \in\{x, y, z\}
$$

and then proceeding by induction in $\bar{\tau}=t-1, \ldots, 1$. The basis of the induction is given by Eq. (D6), so we just need to prove the inductive step. Assuming Eq. (D7) and proceeding as in Eq. (D4), we can show that $A$ commutes also with

$U_{\nu, \phi} e^{-i h M_{\nu}^{z}} e^{i(\pi / 4) M_{\nu}^{x}}\left(\prod_{\tau=\bar{\tau}+1}^{t} \sigma_{\nu, \tau}^{x}\right) e^{-i(\pi / 4) M_{\nu}^{x}} e^{i h M_{\nu}^{z}} U_{\nu, \phi}^{\dagger}=\left(\prod_{\tau=\bar{\tau}+1}^{t}-i \sigma_{\nu, \tau-1}^{z} \sigma_{\nu, \tau}^{z} e^{i 2 h \sigma_{\nu, \tau}^{z}} \sigma_{\nu, \tau}^{x}\right)=-i^{t-\bar{\tau}} \sigma_{\nu, \bar{\tau}}^{z} \sigma_{\nu, t}^{z}\left(\prod_{j=\bar{\tau}+1}^{t} e^{i 2 h \sigma_{\nu, \tau}^{z}} \sigma_{\nu, \tau}^{x}\right)$

The inductive hypothesis (D7) then implies

$$
\left[A, \sigma_{\nu, \bar{\tau}}^{z}\right]=0 .
$$

Reasoning now as in Eq. (D4), we then have that $A$ also commutes with

$$
\begin{gathered}
e^{-i(\pi / 4) M_{\nu}^{x}} e^{i h M_{\nu}^{z}} U_{\nu, \phi}^{\dagger} \sigma_{\nu, \bar{\tau}}^{z} U_{\nu, \phi} e^{-i h M_{\nu}^{z}} e^{i(\pi / 4) M_{\nu}^{x}} \\
=e^{-i(\pi / 4) M_{\nu}^{x}} \sigma_{\nu, \bar{\tau}}^{z} e^{i(\pi / 4) M_{\nu}^{x}}=-\sigma_{\nu, \bar{\tau}}^{y}, \\
e^{-i(\pi / 4) M_{\nu}^{x}} e^{i h M_{\nu}^{z}} U_{\nu, \phi}^{\dagger} \sigma_{\nu, \bar{\tau}}^{z} U_{\nu, \phi} e^{-i h M_{\nu}^{z}} e^{i(\pi / 4) M_{\nu}^{x}} \sigma_{\nu, \bar{\tau}}^{z} \\
=e^{-i(\pi / 4) M_{\nu}^{x}} \sigma_{\nu, \bar{\tau}}^{z} e^{i(\pi / 4) M_{\nu}^{x}} \sigma_{\nu, \bar{\tau}}^{z}=-i \sigma_{\nu, \bar{\tau}}^{x} .
\end{gathered}
$$

So, we have

$$
\begin{aligned}
{\left[A, \sigma_{\nu, \tau}^{a}\right] } & =0, \quad a \in\{x, y, z\}, \\
\tau & \in\{1, \ldots, t\}, \quad \nu \in\{1, \ldots, n\} .
\end{aligned}
$$

This concludes the proof.

\section{APPENDIX E: PROOF OF EQS. (86) AND (87)}

Let us start by proving Eq. (86). First, we note

$$
\prod_{\nu=1}^{n} O_{\nu} \otimes O_{\nu}^{*}|\mathbb{1}\rangle=|\mathbb{1}\rangle
$$

This is explicitly proven as follows:

$$
\begin{aligned}
\prod_{\nu=1}^{n} O_{\nu} \otimes O_{\nu}^{*}|\mathbb{1}\rangle= & \frac{1}{2^{n t / 2}} \sum_{k, m, m^{\prime}}\left\langle m\left|\prod_{\nu=1}^{n} O_{\nu}\right| k\right\rangle\left\langle m^{\prime}\right| \\
& \times \prod_{\nu=1}^{n} O_{\nu}|k\rangle^{*}|m\rangle \otimes\left|m^{\prime}\right\rangle^{*} \\
= & \frac{1}{2^{n t / 2}} \sum_{m, m^{\prime}}\left\langle m\left|\prod_{\nu=1}^{n} O_{\nu} O_{\nu}^{\dagger}\right| m^{\prime}\right\rangle|m\rangle \otimes\left|m^{\prime}\right\rangle^{*} \\
= & \frac{1}{2^{n t / 2}} \sum_{m}|m\rangle \otimes|m\rangle^{*}=|\mathbb{1}\rangle .
\end{aligned}
$$


Here we use Eq. (74) and the fact that $O_{\nu}$ is unitary. Second, we observe that from the definition of $O_{\nu}$, it directly follows

$$
\mathbb{P}\left(\prod_{\nu=1}^{n} O_{\nu} \otimes O_{\nu}^{*}\right) \mathbb{P}^{\dagger}=\prod_{\nu=1}^{n} O_{\nu} \otimes O_{\nu}^{*}
$$

Combining Eqs. (E3) and (E1), we then have

$$
\begin{aligned}
\prod_{\nu=1}^{n} O_{\nu} \otimes O_{\nu}^{*}|\Psi\rangle & =\prod_{\nu=1}^{n} O_{\nu} \otimes O_{\nu}^{*} \mathbb{P}|\mathbb{1}\rangle \\
& =\mathbb{P} \prod_{\nu=1}^{n} O_{\nu} \otimes O_{\nu}^{*}|\mathbb{1}\rangle=|\Psi\rangle .
\end{aligned}
$$

So, we proved Eq. (86) for any $O_{\nu}$ acting nontrivially as the unitary operator $O$ only on the $\nu$ th copy of $\mathcal{H}_{t}$ in $\mathcal{H}_{n t}$. The relation (87) follows immediately by taking the adjoint of

$$
\prod_{\nu=1}^{n} O_{\nu}^{\dagger} \otimes O_{\nu}^{\dagger *}|\Psi\rangle=|\Psi\rangle
$$

\section{APPENDIX F: PROOF OF PROPERTY (84)}

In this Appendix, we prove Eq. (84).

Proof.-Defining

$$
\begin{aligned}
& \mathbb{J}_{\nu, \tau}= \exp \left[-i \frac{\pi}{4} \sigma_{\nu, \tau+1}^{z} \sigma_{\nu, \tau}^{z}\right] \otimes \exp \left[i \frac{\pi}{4} \sigma_{\nu, \tau+1}^{z} \sigma_{\nu, \tau}^{z}\right], \\
& \tau \in\{1, \ldots, t-1\}, \quad \mathbb{J}_{\nu, \tau}=\mathbb{1}, \quad \tau \leq 0, \quad(\mathrm{~F} 1) \\
& \mathbb{Z}_{\nu, \tau}^{h}=\exp \left[-i h \sigma_{\nu, \tau}^{z}\right] \otimes \exp \left[i h \sigma_{\nu, \tau}^{z}\right], \\
& \tau \in\{1, \ldots, t\}, \quad \mathbb{Z}_{\nu, \tau}^{h}=\mathbb{1}, \quad \tau \leq 0, \quad(\mathrm{~F} 2) \\
& \mathbb{X}_{\nu, \tau}=\exp \left[i \frac{\pi}{4} \sigma_{\nu, \tau}^{x}\right] \otimes \exp \left[-i \frac{\pi}{4} \sigma_{\nu, \tau}^{x}\right], \quad \tau \in\{1, \ldots, t\}, \\
& \mathbb{X}_{\nu, \tau}=\mathbb{1}, \quad \tau \leq 0,
\end{aligned}
$$

we can rewrite the lhs of Eq. (84) as follows:

$$
\begin{aligned}
& \left\langle\Psi\left|\prod_{j=1}^{N} \mathbb{T}_{\frac{\pi}{2}, \phi_{j}}\left[h_{j}\right]\right| \Psi\right\rangle \\
& \quad=\left\langle\Psi\left|\prod_{\nu=1}^{n}\left[\prod_{j=1}^{N}\left(\mathbb{G}_{\nu, t}^{z} \mathbb{Z}_{\nu, 1}^{\phi_{j} / 2} \prod_{\tau=1}^{t-1} \mathbb{J}_{\nu, \tau} \prod_{\tau=1}^{t} \mathbb{Z}_{\nu, \tau}^{h_{j}} \prod_{\tau=1}^{t} \mathbb{X}_{\nu, \tau}\right)\right]\right| \Psi\right\rangle .
\end{aligned}
$$

To simplify this expression, we proceed as follows. First, we commute every possible $\mathbb{J}_{\nu, \tau}, \mathbb{Z}_{\nu, \tau}, \mathbb{X}_{\nu, \tau}$ to the left by using the following commutation relations

$$
\begin{aligned}
& \mathbb{J}_{\nu, \tau} \mathbb{Z}_{\nu, \tau^{\prime}}^{h}=\mathbb{Z}_{\nu, \tau^{\prime}}^{h} \mathbb{J}_{\nu, \tau}, \quad \forall \tau, \tau^{\prime}, \\
& \mathbb{J}_{\nu, \tau} \mathbb{X}_{\nu, \tau^{\prime}}=\mathbb{X}_{\nu, \tau^{\prime}} \mathbb{J}_{\nu, \tau}, \quad \tau^{\prime} \neq \tau, \tau+1, \\
& \mathbb{X}_{\nu, \tau} \mathbb{Z}_{\nu, \tau^{\prime}}^{h}=\mathbb{Z}_{\nu, \tau^{\prime}}^{h} \mathbb{X}_{\nu, \tau}, \quad \tau^{\prime} \neq \tau, \\
& \mathbb{J}_{\nu, \tau} \mathbb{G}_{\nu, \tau^{\prime}}^{z}=\mathbb{G}_{\nu, \tau^{\prime}}^{z} \mathbb{J}_{\nu, \tau}, \quad \forall \tau^{\prime}, \tau, \\
& \mathbb{Z}_{\nu, \tau}^{h} \mathbb{G}_{\nu, \tau^{\prime}}^{z}=\mathbb{G}_{\nu, \tau^{\prime}}^{z} \mathbb{Z}_{\nu, \tau}^{h}, \quad \forall \tau^{\prime}, \tau, \\
& \mathbb{X}_{\nu, \tau} \mathbb{G}_{\nu, \tau^{\prime}}^{z}=\mathbb{G}_{\nu, \tau^{\prime}}^{z} \mathbb{X}_{\nu, \tau}, \quad \tau^{\prime} \neq \tau .
\end{aligned}
$$

Then we use

$$
\begin{aligned}
& \langle\Psi| \prod_{\nu=1}^{n} \mathbb{J}_{\nu, \tau}=\langle\Psi|, \\
& \langle\Psi| \prod_{\nu=1}^{n} \mathbb{X}_{\nu, \tau^{\prime}}=\langle\Psi|, \\
& \langle\Psi| \prod_{\nu=1}^{n} \mathbb{Z}_{\nu, \tau^{\prime}}^{h}=\langle\Psi|,
\end{aligned}
$$

which follow from Eq. (86). Finally, using also

$$
\begin{aligned}
& \prod_{\nu=1}^{n} \mathbb{J}_{\nu, \tau}|\Psi\rangle=|\Psi\rangle \\
& \prod_{\nu=1}^{n} \mathbb{X}_{\nu, \tau^{\prime}}|\Psi\rangle=|\Psi\rangle \\
& \prod_{\nu=1}^{n} \mathbb{Z}_{\nu, \tau^{\prime}}^{h}|\Psi\rangle=|\Psi\rangle
\end{aligned}
$$

following from Eq. (87), on the rightmost term in the product over $j$ we find

$$
\left\langle\Psi\left|\prod_{j=1}^{N} \mathbb{T}_{\frac{\pi}{4}, \phi_{j}}\left[h_{j}\right]\right| \Psi\right\rangle=\left\langle\Psi\left|\prod_{\nu=1}^{n}\left[\prod_{j=1}^{N-1} \mathbb{A}_{\nu, j}\right]\right| \Psi\right\rangle,
$$

where we define

$$
\begin{gathered}
\mathbb{A}_{\nu, j}=\mathbb{G}_{\nu, t}^{z} \prod_{\tau=t-j+1}^{t-1} \mathbb{J}_{\nu, \tau} \prod_{\tau=t-j+2}^{t} \tilde{\mathbb{Z}}_{\nu, \tau}^{h_{j}, \phi_{j}} \prod_{\tau=t-j+1}^{t} \mathbb{X}_{\nu, \tau} \mathbb{G}_{\nu, t}^{z}, \\
\tilde{\mathbb{Z}}_{\nu, \tau}^{h, \phi}=\mathbb{Z}_{\nu, \tau}^{h+(\phi / 2) \delta_{\tau, 1}} .
\end{gathered}
$$

Using 


$$
\begin{aligned}
& \mathbb{G}_{\nu, \tau}^{z} \mathbb{X}_{\nu, \tau} \mathbb{G}_{\nu, \tau}^{z}=\mathbb{G}_{\nu, \tau}^{z}\left[\mathbb{G}_{\nu, \tau}^{x}+\frac{i}{2}\left[\sigma_{\nu, \tau}^{x} \otimes \mathbb{1}-\mathbb{1} \otimes \sigma_{\nu, \tau}^{x}\right]\right] \mathbb{G}_{\nu, \tau}^{z} \\
& =\mathbb{G}_{\nu, \tau}^{z}\left[\mathbb{G}_{\nu, \tau}^{z} \mathbb{G}_{\nu, \tau}^{x}+\frac{i}{4}\left[\mathbb{1}-\sigma_{\nu, \tau}^{z} \otimes \sigma_{\nu, \tau}^{z}\right]\left[\sigma_{\nu, \tau}^{x} \otimes \mathbb{1}-\mathbb{1} \otimes \sigma_{\nu, \tau}^{x}\right]\right]=\mathbb{G}_{\nu, \tau}^{z} \mathbb{G}_{\nu, \tau}^{x} \mathbb{G}_{\nu, \tau}^{z}=\mathbb{G}_{\nu, \tau}^{z} \mathbb{G}_{\nu, \tau}^{x},
\end{aligned}
$$

we can rewrite Eq. (F18) as follows:

$$
\mathbb{A}_{\nu, j}=\mathbb{G}_{\nu, t}^{z} \mathbb{J}_{\nu, t-1} \mathbb{G}_{\nu, t}^{x} \prod_{\tau=t-j+1}^{t-2} \mathbb{J}_{\nu, \tau} \prod_{\tau=t-j+2}^{t-1} \tilde{\mathbb{Z}}_{\nu, \tau}^{h_{j}, \phi_{j}} \prod_{\tau=t-j+1}^{t-1} \mathbb{X}_{\nu, \tau}
$$

We now make use the following lemma proven in Appendix F 1 to simplify the products of the $\mathbb{A}_{\nu, j}$ 's

Lemma 1.

$$
\begin{aligned}
\mathbb{A}_{\nu, 1} \cdots \mathbb{A}_{\nu, 2 n} & =\mathbb{J}_{\nu, t-1} \prod_{j=0}^{n-1}\left[\mathbb{G}_{\nu, t-j}^{z} \mathbb{G}_{\nu, t-j}^{x}\right] \mathbb{G}_{\nu, t-n}^{z} \mathbb{X}_{\nu, t-n} \prod_{j=n}^{2 n-2}\left[\prod_{\tau=t-1-j}^{t-2 n+j} \mathbb{J}_{\nu, \tau} \prod_{\tau=t-j}^{t-2 n+j} \tilde{\mathbb{Z}}_{\nu, \tau}^{h_{j+2}, \phi_{j+2}} \prod_{\tau=t-1-j}^{t-2 n+j+1} \mathbb{X}_{\nu, \tau}\right], \\
\mathbb{A}_{\nu, 1} \cdots \mathbb{A}_{\nu, 2 n+1} & =\mathbb{J}_{\nu, t-1} \prod_{j=0}^{n}\left[\mathbb{G}_{\nu, t-j}^{z} \mathbb{G}_{\nu, t-j}^{x}\right] \prod_{j=n}^{2 n-1}\left[\prod_{\tau=t-1-j}^{t-2 n-1+j} \mathbb{J}_{\nu, \tau} \prod_{\tau=t-j}^{t-2 n+j-1} \tilde{\mathbb{Z}}_{\nu, \tau}^{h_{j+2}, \phi_{j+2}} \prod_{\tau=t-1-j}^{t-2 n+j} \mathbb{X}_{\nu, \tau}\right], \quad n \geq 1 .
\end{aligned}
$$

Using now Eqs. (F11) and (F14)-(F16), we have

$$
\left\langle\Psi\left|\prod_{j=1}^{N} \mathbb{T}_{\pi / 4, \phi_{j}}\left[h_{j}\right]\right| \Psi\right\rangle=\left\langle\Psi\left|\prod_{\nu=1}^{n}\left[\prod_{j=0}^{\lfloor N / 2\rfloor-1}\left[\mathbb{G}_{\nu, t-j}^{z} \mathbb{G}_{\nu, t-j}^{x}\right]\left[\mathbb{G}_{\nu, t-\lfloor N / 2\rfloor}^{z}\right] \bmod (N, 2)\right]\right| \Psi\right\rangle,
$$

which concludes the proof.

\section{Proof of Lemma 1}

Here we prove Lemma 1.

Proof.-We proceed by induction in the number of terms in the products of the $\mathbb{A}_{\nu, j}$ 's. First, we establish the basis. We begin by computing

$$
\mathbb{A}_{\nu, 1} \mathbb{A}_{\nu, 2}=\mathbb{G}_{\nu, t}^{z} \rrbracket_{\nu, t-1} \mathbb{G}_{\nu, t}^{x} \mathbb{G}_{\nu, t}^{z} \mathbb{J}_{\nu, t-1} \mathbb{G}_{\nu, t}^{x} \mathbb{\mathbb { V }}_{\nu, t-1}=\mathbb{J}_{\nu, t-1} \mathbb{G}_{\nu, t}^{z} \mathbb{G}_{\nu, t}^{x} \mathbb{G}_{\nu, t-1}^{z} \mathbb{X}_{\nu, t-1}
$$

where we use

$$
\begin{aligned}
& \mathbb{G}_{\nu, \tau}^{z} \mathbb{G}_{\nu, \tau}^{x} \mathbb{J}_{\nu, \tau-1} \mathbb{G}_{\nu, \tau}^{x}=\mathbb{G}_{\nu, \tau}^{z} \mathbb{G}_{\nu, \tau}^{x}\left[\mathbb{G}_{\nu, \tau-1}^{z}+\frac{i}{2}\left[\sigma_{\nu, \tau-1}^{z} \otimes \sigma_{\nu, \tau}^{z}-\sigma_{\nu, \tau}^{z} \otimes \sigma_{\nu, \tau-1}^{z}\right]\right] \mathbb{G}_{\nu, t}^{x} \\
& =\mathbb{G}_{\nu, \tau}^{z} \mathbb{G}_{\nu, \tau}^{x}\left[\mathbb{G}_{\nu, \tau}^{x} \mathbb{G}_{\nu, \tau-1}^{z}+\frac{i}{4}\left[\mathbb{1}-\sigma_{\nu, \tau}^{x} \otimes \sigma_{\nu, \tau}^{x}\right]\left[\sigma_{\nu, \tau-1}^{z} \otimes \sigma_{\nu, \tau}^{z}-\sigma_{\nu, \tau}^{z} \otimes \sigma_{\nu, \tau-1}^{z}\right]\right]=\mathbb{G}_{\nu, \tau}^{z} \mathbb{G}_{\nu, \tau}^{x} \mathbb{G}_{\nu, \tau-1}^{z} .
\end{aligned}
$$

We see that Eq. (F25) agrees with Eq. (F22) for $n=1$. We then compute

$$
\begin{aligned}
& \mathbb{A}_{\nu, 1} \mathbb{A}_{\nu, 2} \mathbb{A}_{\nu, 3}=\mathbb{G}_{\nu, t}^{z} \mathbb{\rrbracket}_{\nu, t-1} \mathbb{G}_{\nu, t}^{x} \mathbb{G}_{\nu, t}^{z} \mathbb{\Xi}_{\nu, t-1} \mathbb{G}_{\nu, t}^{x} \mathbb{X}_{\nu, t-1} \mathbb{G}_{\nu, t}^{z} \rrbracket_{\nu, t-1} \mathbb{G}_{\nu, t}^{x} \rrbracket_{\nu, t-2} \tilde{\mathbb{Z}}_{\nu, t-1}^{h_{3}, \phi_{3}} \mathbb{X}_{\nu, t-2} \mathbb{X}_{\nu, t-1} \\
& =\mathbb{J}_{\nu, t-1} \mathbb{G}_{\nu, t}^{z} \mathbb{G}_{\nu, t}^{x} \mathbb{G}_{\nu, t-1}^{z} \mathbb{X}_{\nu, t-1} \mathbb{G}_{\nu, t}^{z} \mathbb{V}_{\nu, t-1} \mathbb{G}_{\nu, t}^{x} \mathbb{\Xi}_{\nu, t-2} \tilde{\mathbb{Z}}_{\nu, t-1}^{h_{3}, \phi_{3}} \mathbb{X}_{\nu, t-2} \mathbb{X}_{\nu, t-1} \\
& =\mathbb{J}_{\nu, t-1} \mathbb{G}_{\nu, t}^{z} \mathbb{G}_{\nu, t}^{x} \mathbb{G}_{\nu, t-1}^{z} \mathbb{X}_{\nu, t-1} \mathbb{G}_{\nu, t-1}^{z} \mathbb{U}_{\nu, t-2} \tilde{\mathbb{Z}}_{\nu, t-1}^{h_{3}, \phi_{3}} \mathbb{X}_{\nu, t-2} \mathbb{X}_{\nu, t-1} \\
& =\mathbb{J}_{\nu, t-1} \mathbb{G}_{\nu, t}^{z} \mathbb{G}_{\nu, t}^{x} \mathbb{G}_{\nu, t-1}^{z} \mathbb{G}_{\nu, t-1}^{x} \mathbb{J}_{\nu, t-2} \tilde{\mathbb{Z}}_{\nu, t-1}^{h_{3}, \phi_{3}} \mathbb{X}_{\nu, t-2} \mathbb{X}_{\nu, t-1} \\
& =\mathbb{J}_{\nu, t-1} \mathbb{G}_{\nu, t}^{z} \mathbb{G}_{\nu, t}^{x} \mathbb{G}_{\nu, t-1}^{z} \mathbb{G}_{\nu, t-1}^{x} \mathbb{J}_{\nu, t-2} \mathbb{X}_{\nu, t-2} \mathbb{X}_{\nu, t-1}
\end{aligned}
$$

In the last step, we use 


$$
\mathbb{G}_{\nu, \tau}^{z} \tilde{\mathbb{Z}}_{\nu, \tau}^{h, \phi}=\mathbb{G}_{\nu, \tau}^{z}
$$

Since Eq. (F27) agrees with Eq. (F23) for $n=1$, we successfully establish the basis for the inductive procedure. To conclude, we need to prove that

(i) if Eq. (F22) holds for $n$, then Eq. (F23) holds for $n$;

(ii) if Eq. (F23) holds for $n$, then Eq. (F22) holds for $n+1$.

Let us prove (i). Assuming Eq. (F22), we have

$$
\begin{aligned}
\mathbb{A}_{\nu, 1} \ldots \mathbb{A}_{\nu, 2 n} \mathbb{A}_{\nu, 2 n+1}= & \mathbb{J}_{\nu, t-1} \prod_{j=0}^{n-1}\left[\mathbb{G}_{\nu, t-j}^{z} \mathbb{G}_{\nu, t-j}^{x}\right] \mathbb{G}_{\nu, t-n}^{z} \mathbb{X}_{\nu, t-n} \prod_{j=n}^{2 n-2}\left[\prod_{\tau=t-1-j}^{t-2 n+j} \mathbb{J}_{\nu, \tau} \prod_{\tau=t-j}^{t-2 n+j} \tilde{\mathbb{Z}}_{\nu, \tau}^{h_{j+2}, \phi_{j+2}} \prod_{\tau=t-1-j}^{t-2 n+j+1} \mathbb{\mathbb { X }}_{\nu, \tau}\right], \\
& \times \mathbb{G}_{\nu, t}^{z} \mathbb{\Xi}_{\nu, t-1} \mathbb{G}_{\nu, t}^{x} \prod_{\tau=t-2 n}^{t-2} \mathbb{J}_{\nu, \tau} \prod_{\tau=t-2 n+1}^{t-1} \tilde{\mathbb{Z}}_{\nu, \tau}^{h_{2 n+1}, \phi_{2 n+1}} \prod_{\tau=t-2 n}^{t-1} \mathbb{X}_{\nu, \tau} \\
= & \mathbb{J}_{\nu, t-1} \prod_{j=0}^{n-1}\left[\mathbb{G}_{\nu, t-j}^{z} \mathbb{G}_{\nu, t-j}^{x}\right] \mathbb{G}_{\nu, t-n}^{z} \mathbb{X}_{\nu, t-n} \prod_{j=n}^{2 n-2}\left[\prod_{\tau=t-1-j}^{t-2 n+j} \mathbb{J}_{\nu, \tau} \prod_{\tau=t-j}^{t-2 n+j} \tilde{\mathbb{Z}}_{\nu, \tau}^{h_{j+2}, \phi_{j+2}} \prod_{\tau=t-1-j}^{t-2 n+j+1} \mathbb{\mathbb { X }}_{\nu, \tau}\right], \\
& \times \mathbb{G}_{\nu, t-1}^{z} \prod_{\tau=t-2 n}^{t-2} \mathbb{J}_{\nu, \tau} \prod_{\tau=t-2 n+1}^{t-1} \tilde{\mathbb{Z}}_{\nu, \tau}^{h_{2 n+1}, \phi_{2 n+1}} \prod_{\tau=t-2 n}^{t-1} \mathbb{X}_{\nu, \tau},
\end{aligned}
$$

where we use that $\mathbb{G}_{\nu, t}^{x}$ commutes with all the terms on the first line to bring it close to $\mathbb{J}_{\nu, t-1}$. Then we employ Eq. (F26). Moving the projector $\mathbb{G}_{\nu, t-1}^{z}$ on the second line to the left and using multiple times Eqs. (F20) and (F26), we have

$$
\begin{aligned}
\mathbb{A}_{\nu, 1} \ldots \mathbb{A}_{\nu, 2 n} \mathbb{A}_{\nu, 2 n+1}= & \mathbb{J}_{\nu, t-1} \prod_{j=0}^{n-1}\left[\mathbb{G}_{\nu, t-j}^{z} \mathbb{G}_{\nu, t-j}^{x}\right] \mathbb{G}_{\nu, t-n}^{z} \mathbb{X}_{\nu, t-n} \mathbb{G}_{\nu, t-n}^{z} \prod_{j=n}^{2 n-2}\left[\prod_{\tau=t-1-j}^{t-2 n+j-1} \mathbb{J}_{\nu, \tau} \prod_{\tau=t-j}^{t-2 n+j-1} \tilde{\mathbb{Z}}_{\nu, \tau}^{h_{j+2}, \phi_{j+2}} \prod_{\tau=t-1-j}^{t-2 n+j} \mathbb{X}_{\nu, \tau}\right], \\
& \times \prod_{\tau=t-2 n}^{t-2} \mathbb{J}_{\nu, \tau} \prod_{\tau=t-2 n+1}^{t-1} \tilde{\mathbb{Z}}_{\nu, \tau}^{h_{2 n+1}, \phi_{2 n+1}} \prod_{\tau=t-2 n}^{t-1} \mathbb{\mathbb { V }}_{\nu, \tau} \\
= & \mathbb{J}_{\nu, t-1} \prod_{j=0}^{n-1}\left[\mathbb{G}_{\nu, t-j}^{z} \mathbb{G}_{\nu, t-j}^{x}\right] \mathbb{G}_{\nu, t-n}^{z} \mathbb{G}_{\nu, t-n}^{x} \prod_{j=n}^{2 n-2}\left[\prod_{\tau=t-1-j}^{t-2 n+j-1} \mathbb{J}_{\nu, \tau} \prod_{\tau=t-j}^{t-2 n+j-1} \tilde{\mathbb{Z}}_{\nu, \tau}^{h_{j+2}, \phi_{j+2}} \prod_{\tau=t-1-j}^{t-2 n+j} \mathbb{X}_{\nu, \tau}\right], \\
& \times \prod_{\tau=t-2 n}^{t-2} \mathbb{J}_{\nu, \tau} \prod_{\tau=t-2 n+1}^{t-1} \tilde{\mathbb{Z}}_{\nu, \tau}^{h_{2 n+1}, \phi_{2 n+1}} \prod_{\tau=t-2 n}^{t-1} \mathbb{\mathbb { X }}_{\nu, \tau} \\
= & \mathbb{J}_{\nu, t-1} \prod_{j=0}^{n}\left[\mathbb{G}_{\nu, t-j}^{z} \mathbb{G}_{\nu, t-j}^{x}\right] \prod_{j=n}^{2 n-1}\left[\prod_{\tau=t-1-j}^{t-2 n+j-1} \mathbb{J}_{\nu, \tau} \prod_{\tau=t-j}^{t-2 n+j-1} \tilde{\mathbb{Z}}_{\nu, \tau}^{h_{j+2}, \phi_{j+2}} \prod_{\tau=t-1-j}^{t-2 n+j} \mathbb{X}_{\nu, \tau}\right],
\end{aligned}
$$

which is exactly Eq. (F23). Let us now prove (ii). Assuming Eq. (F23), we have

$$
\begin{aligned}
\mathbb{A}_{\nu, 1} \ldots \mathbb{A}_{\nu, 2 n+1} \mathbb{A}_{\nu, 2 n+2}= & \mathbb{J}_{\nu, t-1} \prod_{j=0}^{n}\left[\mathbb{G}_{\nu, t-j}^{z} \mathbb{G}_{\nu, t-j}^{x}\right] \prod_{j=n}^{2 n-1}\left[\prod_{\tau=t-1-j}^{t-2 n-1+j} \mathbb{J}_{\nu, \tau} \prod_{\tau=t-j}^{t-2 n+j-1} \tilde{\mathbb{Z}}_{\nu, \tau}^{h_{j+2}, \phi_{j+2}} \prod_{\tau=t-1-j}^{t-2 n+j} \mathbb{X}_{\nu, \tau}\right] \\
& \times \mathbb{G}_{\nu, t}^{z} \mathbb{J}_{\nu, t-1} \mathbb{G}_{\nu, t}^{x} \prod_{\tau=t-2 n-1}^{t-2} \mathbb{J}_{\nu, \tau} \prod_{\tau=t-2 n}^{t-1} \tilde{\mathbb{Z}}_{\nu, \tau}^{h_{2 n+2}, \phi_{2 n+2}} \prod_{\tau=t-2 n-1}^{t-1} \mathbb{\mathbb { V }}_{\nu, \tau} \\
= & \mathbb{J}_{\nu, t-1} \prod_{j=0}^{n}\left[\mathbb{G}_{\nu, t-j}^{z} \mathbb{G}_{\nu, t-j}^{x}\right] \prod_{j=n}^{2 n-1}\left[\prod_{\tau=t-1-j}^{t-2 n-1+j} \mathbb{J}_{\nu, \tau} \prod_{\tau=t-j}^{t-2 n+j-1} \tilde{\mathbb{Z}}_{\nu, \tau}^{h_{j+2}, \phi_{j+2}} \prod_{\tau=t-1-j}^{t-2 n+j} \mathbb{\mathbb { V }}_{\nu, \tau}\right] \\
& \times \mathbb{G}_{\nu, t-1}^{z} \prod_{\tau=t-2 n-1}^{t-2} \mathbb{J}_{\nu, \tau} \prod_{\tau=t-2 n}^{t-1} \tilde{\mathbb{Z}}_{\nu, \tau}^{h_{2 n+2}, \phi_{2 n+2}} \prod_{\tau=t-2 n-1}^{t-1} \mathbb{X}_{\nu, \tau},
\end{aligned}
$$

where we again use that $\mathbb{G}_{\nu, t}^{x}$ commutes with all the terms on the first line to bring it close to $\mathbb{J}_{\nu, t-1}$ and employ Eq. (F26). Moving now the projector $\mathbb{G}_{\nu, t-1}^{z}$ on the second line to the left and using Eqs. (F20) and (F26), we have 


$$
\begin{aligned}
\mathbb{A}_{\nu, 1} \cdots \mathbb{A}_{\nu, 2 n+2}= & \mathbb{J}_{\nu, t-1} \prod_{j=0}^{n}\left[\mathbb{G}_{\nu, t-j}^{z} \mathbb{G}_{\nu, t-j}^{x}\right] \mathbb{G}_{\nu, t-n-1}^{z} \mathbb{X}_{\nu, t-n-1} \prod_{j=n}^{2 n-1}\left[\prod_{\tau=t-1-j}^{t-2 n-2+j} \mathbb{J}_{\nu, \tau} \prod_{\tau=t-j}^{t-2 n+j-2} \tilde{\mathbb{Z}}_{\nu, \tau}^{h_{j+2}, \phi_{j+2}} \prod_{\tau=t-1-j}^{t-2 n-1+j} \mathbb{X}_{\nu, \tau}\right] \\
& \times \prod_{\tau=t-2 n-1}^{t-2} \mathbb{J}_{\nu, \tau} \prod_{\tau=t-2 n}^{t-1} \tilde{\mathbb{Z}}_{\nu, \tau}^{h_{2 n+2}, \phi_{2 n+2}} \prod_{\tau=t-2 n-1}^{t-1} \mathbb{X}_{\nu, \tau} \\
= & \mathbb{J}_{\nu, t-1} \prod_{j=0}^{n}\left[\mathbb{G}_{\nu, t-j}^{z} \mathbb{G}_{\nu, t-j}^{x}\right] \mathbb{G}_{\nu, t-n-1}^{z} \mathbb{X}_{\nu, t-n-1} \prod_{j=n}^{2 n}\left[\prod_{\tau=t-1-j}^{t-2 n-2+j} \mathbb{J}_{\nu, \tau} \prod_{\tau=t-j}^{t-2 n+j-2} \tilde{\mathbb{Z}}_{\nu, \tau}^{h_{j+2}, \phi_{j+2}} \prod_{\tau=t-1-j}^{t-2 n-1+j} \mathbb{X}_{\nu, \tau}\right]
\end{aligned}
$$

which is exactly Eq. (F22) for $n+1$. This concludes the proof.

[1] A. Einstein, B. Podolsky, and N. Rosen, Can QuantumMechanical Description of Physical Reality Be Considered Complete?, Phys. Rev. 47, 777 (1935).

[2] J. S. Bell, On the Problem of Hidden Variables in Quantum Mechanics, Rev. Mod. Phys. 38, 447 (1966).

[3] L. Amico, R. Fazio, A. Osterloh, and V. Vedral, Entanglement in Many-Body Systems, Rev. Mod. Phys. 80, 517 (2008).

[4] N. Laflorencie, Quantum Entanglement in Condensed Matter Systems, Phys. Rep. 646, 1 (2016).

[5] N. Schuch, M. M. Wolf, F. Verstraete, and J. I. Cirac, Entropy Scaling and Simulability by Matrix Product States, Phys. Rev. Lett. 100, 030504 (2008).

[6] N. Schuch, M. M. Wolf, K. G. H. Vollbrecht, and J. I. Cirac, On Entropy Growth and the Hardness of Simulating Time Evolution, New J. Phys. 10, 033032 (2008).

[7] A. Perales and G. Vidal, Entanglement Growth and Simulation Efficiency in One-Dimensional Quantum Lattice Systems, Phys. Rev. A 78, 042337 (2008).

[8] P. Hauke, F. M. Cucchietti, L. Tagliacozzo, I. Deutsch, and M. Lewenstein, Can One Trust Quantum Simulators?, Prog. Phys. 75, 082401 (2012).

[9] J. Dubail, Entanglement Scaling of Operators: A Conformal Field Theory Approach, with a Glimpse of Simulability of Long-Time Dynamics in $1+1 d$, J. Phys. A 50, 234001 (2017).

[10] P. Calabrese, Entanglement and Thermodynamics in NonEquilibrium Isolated Quantum Systems, Physica (Amsterdam) 504A, 31 (2018).

[11] J. M. Deutsch, H. Li, and A. Sharma, Microscopic Origin of Thermodynamic Entropy in Isolated Systems, Phys. Rev. E 87, 042135 (2013).

[12] W. Beugeling, A. Andreanov, and M. Haque, Global Characteristics of All Eigenstates of Local Many-Body Hamiltonians: Participation Ratio and Entanglement Entropy, J. Stat. Mech. (2015) P02002.

[13] V. Gurarie, Global Large Time Dynamics and the Generalized Gibbs Ensemble, J. Stat. Mech. (2013) P02014.

[14] L. F. Santos, A. Polkovnikov, and M. Rigol, Entropy of Isolated Quantum Systems after a Quench, Phys. Rev. Lett. 107, 040601 (2011).

[15] P. Calabrese and J. Cardy, Evolution of Entanglement Entropy in One-Dimensional Systems, J. Stat. Mech. (2005) P04010.
[16] G. De Chiara, S. Montangero, P. Calabrese, and R. Fazio, Entanglement Entropy Dynamics of Heisenberg Chains, J. Stat. Mech. (2006) P03001.

[17] M. Fagotti and P. Calabrese, Evolution of Entanglement Entropy Following a Quantum Quench: Analytic Results for the XY Chain in a Transverse Magnetic Field, Phys. Rev. A 78, 010306(R).

[18] V. Eisler and I. Peschel, Entanglement in a Periodic Quench, Ann. Phys. (Berlin) 17, 410 (2008).

[19] M. G. Nezhadhaghighi and M. A. Rajabpour, Entanglement Dynamics in Short- and Long-Range Harmonic Oscillators, Phys. Rev. B 90, 205438 (2014).

[20] L. Bucciantini, M. Kormos, and P. Calabrese, Quantum Quenches from Excited States in the Ising Chain, J. Phys. A 47, 175002 (2014).

[21] E. Bianchi, L. Hackl, and N. Yokomizo, Linear Growth of the Entanglement Entropy and the Kolmogorov-Sinai Rate, J. High Energy Phys. 03 (2018) 25.

[22] L. Hackl, E. Bianchi, R. Modak, and M. Rigol, Entanglement Production in Bosonic Systems: Linear and Logarithmic Growth, Phys. Rev. A 97, 032321 (2018).

[23] M. Fagotti and M. Collura, Universal Prethermalization Dynamics of Entanglement Entropies after a Global Quench, arXiv:1507.02678.

[24] A. S. Buyskikh, M. Fagotti, J. Schachenmayer, F. Essler, and A. J. Daley, Entanglement Growth and Correlation Spreading with Variable-Range Interactions in Spin and Fermionic Tunneling Models, Phys. Rev. A 93, 053620 (2016).

[25] J. S. Cotler, M. P. Hertzberg, M. Mezei, and M. T. Mueller, Entanglement Growth after a Global Quench in Free Scalar Field Theory, J. High Energy Phys. 11 (2016) 166.

[26] V. Alba and P. Calabrese, Entanglement and Thermodynamics after a Quantum Quench in Integrable Systems, Proc. Natl. Acad. Sci. U.S.A. 114, 7947 (2017); V. Alba and P. Calabrese, Entanglement Dynamics after Quantum Quenches in Generic Integrable Systems, SciPost Phys. 4, 017 (2018).

[27] M. Mestyan, B. Bertini, L. Piroli, and P. Calabrese, Exact Solution for the Quench Dynamics of a Nested Integrable System, J. Stat. Mech. (2017) 083103.

[28] C. P. Moca, M. Kormos, and G. Zarand, Hybrid Semiclassical Theory of Quantum Quenches in One-Dimensional Systems, Phys. Rev. Lett. 119, 100603 (2017).

[29] I. Frerot, P. Naldesi, and T. Roscilde, Multispeed Prethermalization in Quantum Spin Models with PowerLaw Decaying Interactions, Phys. Rev. Lett. 120, 050401 (2018). 
[30] M. Collura, M. Kormos, and G. Takacs, Dynamical Manifestation of the Gibbs Paradox after a Quantum Quench, Phys. Rev. A 98, 053610 (2018).

[31] B. Bertini, E. Tartaglia, and P. Calabrese, Entanglement and Diagonal Entropies after a Quench with No Pair Structure, J. Stat. Mech. (2018) 063104.

[32] A. Bastianello and P. Calabrese, Spreading of Entanglement and Correlations after a Quench with Intertwined Quasiparticles, SciPost Phys. 5, 033 (2018).

[33] H. Liu and S. J. Suh, Entanglement Tsunami: Universal Scaling in Holographic Thermalization, Phys. Rev. Lett. 112, 011601 (2014).

[34] H. Casini, H. Liu, and M. Mezei, Spread of Entanglement and Causality, J. High Energy Phys. 07 (2016) 077.

[35] C. T. Asplund, A. Bernamonti, F. Galli, and T. Hartman, Entanglement Scrambling in $2 d$ Conformal Field Theory, J. High Energy Phys. 09 (2015) 110.

[36] S. Leichenauer and M. Moosa, Entanglement Tsunami in (1 + 1)-Dimensions, Phys. Rev. D 92, 126004 (2015).

[37] V. Alba, Entanglement and Quantum Transport in Integrable Systems, Phys. Rev. B 97, 245135 (2018).

[38] B. Bertini, M. Fagotti, L. Piroli, and P. Calabrese, Entanglement Evolution and Generalised Hydrodynamics: Noninteracting Systems, J. Phys. A 51, 39LT01 (2018).

[39] V. Alba, B. Bertini, and M. Fagotti, Entanglement Evolution and Generalised Hydrodynamics: Interacting Integrable Systems, arXiv:1903.00467.

[40] A. J. Daley, H. Pichler, J. Schachenmayer, and P. Zoller, Measuring Entanglement Growth in Quench Dynamics of Bosons in an Optical Lattice, Phys. Rev. Lett. 109, 020505 (2012).

[41] A. Elben, B. Vermersch, M. Dalmonte, J. I. Cirac, and P. Zoller, Rényi Entropies from Random Quenches in Atomic Hubbard and Spin Models, Phys. Rev. Lett. 120, 050406 (2018).

[42] A. Laeuchli and C. Kollath, Spreading of Correlations and Entanglement after a Quench in the One-Dimensional BoseHubbard Model, J. Stat. Mech. (2008) P05018.

[43] H. Kim and D. A. Huse, Ballistic Spreading of Entanglement in a Diffusive Nonintegrable System, Phys. Rev. Lett. 111, 127205 (2013).

[44] R. Pal and A. Lakshminarayan, Entangling Power of TimeEvolution Operators in Integrable and Nonintegrable Many-Body Systems, Phys. Rev. B 98, 174304 (2018).

[45] A. M. Kaufman, M. E. Tai, A. Lukin, M. Rispoli, R. Schittko, P. M. Preiss, and M. Greiner, Quantum Thermalization through Entanglement in an Isolated Many-Body System, Science 353, 794 (2016).

[46] M. Žnidarič, T. Prosen, and P. Prelovšek, Many-Body Localization in the Heisenberg XXZ Magnet in a Random Field, Phys. Rev. B 77, 064426 (2008).

[47] M. Kormos, M. Collura, G. Takács, and P. Calabrese, RealTime Confinement Following a Quantum Quench to a NonIntegrable Model, Nat. Phys. 13, 246 (2017).

[48] A. Nahum, J. Ruhman, and D. A. Huse, Dynamics of Entanglement and Transport in One-Dimensional Systems with Quenched Randomness, Phys. Rev. B 98, 035118 (2018).

[49] R. Nandkishore and D. A. Huse, Many-Body Localization and Thermalization in Quantum Statistical Mechanics, Annu. Rev. Condens. Matter Phys. 6, 15 (2015).
[50] A. Nahum, J. Ruhman, S. Vijay, and J. Haah, Quantum Entanglement Growth under Random Unitary Dynamics, Phys. Rev. X 7, 031016 (2017).

[51] C. von Keyserlingk, T. Rakovszky, F. Pollmann, and S. Sondhi, Operator Hydrodynamics, OTOCs, and Entanglement Growth in Systems without Conservation Laws, Phys. Rev. X 8, 021013 (2018).

[52] A. Nahum, S. Vijay, and J. Haah, Operator Spreading in Random Unitary Circuits, Phys. Rev. X 8, 021014 (2018).

[53] C. Jonay, D. A. Huse, and A. Nahum, Coarse-Grained Dynamics of Operator and State Entanglement, arXiv:1803 .00089

[54] T. Zhou and A. Nahum, Emergent Statistical Mechanics of Entanglement in Random Unitary Circuits, arXiv:1804 .09737 .

[55] A. Chan, A. De Luca, and J. T. Chalker, Solution of a Minimal Model for Many-Body Quantum Chaos, Phys. Rev. X 8, 041019 (2018).

[56] B. Bertini, P. Kos, and T. Prosen, Exact Spectral Form Factor in a Minimal Model of Many-Body Quantum Chaos, Phys. Rev. Lett. 121, 264101 (2018).

[57] Note that the notions "quantum chaotic" and "ergodic" are equivalent for a quantum many-body system and are used interchangeably in the paper.

[58] T. Prosen, General Relation between Quantum Ergodicity and Fidelity of Quantum Dynamics, Phys. Rev. E 65, 036208 (2002).

[59] T. Prosen, Ruelle Resonances in Quantum Many-Body Dynamics, J. Phys. A 35, L737 (2002).

[60] T. Prosen, Chaos and Complexity of Quantum Motion, J. Phys. A 40, 7881 (2007).

[61] C. Pineda and T. Prosen, Universal and Nonuniversal Level Statistics in a Chaotic Quantum Spin Chain, Phys. Rev. E 76, 061127 (2007).

[62] P. Ponte, Z. Papić, F. Huveneers, and D. A. Abanin, ManyBody Localization in Periodically Driven Systems, Phys. Rev. Lett. 114, 140401 (2015).

[63] R. Moessner and S. L. Sondhi, Equilibration and Order in Quantum Floquet Matter, Nat. Phys. 13, 424 (2017).

[64] G. K. Naik, R. Singh, and S. K. Mishra, Controlled Generation of Genuine Multipartite Entanglement in Floquet Ising Spin Models, Phys. Rev. A 99, 032321 (2019).

[65] I. Bloch, J. Dalibard, and W. Zwerger, Many-Body Physics with Ultracold Gases, Rev. Mod. Phys. 80, 885 (2008).

[66] P. Bordia, H. Lüschen, U. Schneider, M. Knap, and I. Bloch, Periodically Driving a Many-Body Localized Quantum System, Nat. Phys. 13, 460 (2017).

[67] J. Zhang, P. W. Hess, A. Kyprianidis, P. Becker, A. Lee, J. Smith, G. Pagano, I.-D. Potirniche, A. C. Potter, A. Vishwanath, N. Y. Yao, and C. Monroe, Observation of a Discrete Time Crystal, Nature (London) 543, 217 (2017).

[68] Note that for $\theta_{j} \in\{0, \pi\}$ the states (12) are independent of $\boldsymbol{\phi}$. This means that we do not need to specify the value of $\boldsymbol{\phi}$ in Eq. (18).

[69] S. K. Mishra, A. Lakshminarayan, and V. Subrahmanyam, Protocol Using Kicked Ising Dynamics for Generating States with Maximal Multipartite Entanglement, Phys. Rev. A 91, 022318 (2015). 
[70] M. Akila, D. Waltner, B. Gutkin, and T. Guhr, Particle-Time Duality in the Kicked Ising Spin Chain, J. Phys. A 49, 375101 (2016).

[71] F. H. L. Essler and M. Fagotti, Quench Dynamics and Relaxation in Isolated Integrable Quantum Spin Chains, J. Stat. Mech. (2016) 064002.

[72] L. Vidmar and M. Rigol, Generalized Gibbs Ensemble in Integrable Lattice Models, J. Stat. Mech. (2016) 064007.

[73] D. N. Page, Average Entropy of a Subsystem, Phys. Rev. Lett. 71, 1291 (1993).

[74] L. Vidmar and M. Rigol, Entanglement Entropy of Eigenstates of Quantum Chaotic Hamiltonians, Phys. Rev. Lett. 119, 220603 (2017).

[75] S. Sotiriadis, Memory-Preserving Equilibration after a Quantum Quench in a One-Dimensional Critical Model, Phys. Rev. A 94, 031605(R) (2016).

[76] M. Gluza, C. Krumnow, M. Friesdorf, C. Gogolin, and J. Eisert, Equilibration via Gaussification in Fermionic Lattice Systems, Phys. Rev. Lett. 117, 190602 (2016).
[77] Note that the density of free (Bogoliubov) fermions is independent of $\phi$. The $\phi$ dependence of $S_{\theta, \phi}^{(n)}$ (see the inset of Fig. 8) then implies that the latter is not entirely fixed by the fermionic density. This is expected for non-Gaussian states.

[78] M. C. Bañuls, M. B. Hastings, F. Verstraete, and J. I. Cirac, Matrix Product States for Dynamical Simulation of Infinite Chains, Phys. Rev. Lett. 102, 240603 (2009).

[79] Note that $m=J_{\max }-1$, where $J_{\max }$ is the size of the largest Jordan block associated with the eigenvalue 0. For separating states we have $m=2 t$. Our numerical analysis shows that this remains true for all states (12).

[80] M. Moeckel and S. Kehrein, Interaction Quench in the Hubbard Model, Phys. Rev. Lett. 100, 175702 (2008).

[81] B. Bertini, F. H. L. Essler, S. Groha, and N. J. Robinson, Prethermalization and Thermalization in Models with Weak Integrability Breaking, Phys. Rev. Lett. 115, 180601 (2015).

[82] T. Langen, T. Gasenzer, and J. Schmiedmayer, Prethermalization and Universal Dynamics in Near-Integrable Quantum Systems, J. Stat. Mech. (2016) 064009. 